\title{
MARCHENKO EQUATIONS AND NORMING CONSTANTS OF THE MATRIX ZAKHAROV-SHABAT SYSTEM
}

\author{
FRANCESCO DEMONTIS AND CORNELIS VAN DER MEE
}

(communicated by A. Ran)

\begin{abstract}
In this article we derive the Marchenko integral equations for solving the inverse scattering problem for the matrix Zakharov-Shabat system with a potential without symmetry properties and having $L^{1}$ entries under a technical hypothesis preventing the accumulation of discrete eigenvalues on the continuous spectrum. We derive additional symmetry properties in the focusing case. The norming constant matrices appearing as parameter matrices in the Marchenko integral kernels are defined and studied without making any assumptions on the Jordan structure of the matrix Zakharov-Shabat Hamiltonian at the discrete eigenvalues.
\end{abstract}

\section{Introduction}

It is well-known that the inverse scattering problem for the Schrödinger equation on the line has a unique solution if the bound state energies, one of the reflection coefficients, and as many so-called norming constants as there are bound states are given $[8,10,12]$. These norming constants are positive numbers appearing as the products of the constant ratio of the Jost solutions at the bound states (called the dependency constants) and the imaginary parts of the residues of the transmission coefficients at the bound states. They appear as parameters in the integral kernel of the Marchenko integral equation whose solution yields the potential. A similar result is true for the focusing (matrix) Zakharov-Shabat system $[18,15]$. The theory of dependency and norming constants and their appearance in the integral kernels of the Marchenko integral equations solving the inverse scattering problem is particularly straightforward if the discrete eigenvalues of the underlying Hamiltonian are geometrically and algebraically simple.

Generalizations of dependency and norming constants require a careful study of the Jordan structure of the discrete eigenvalues. This was done for the zero eigenvalue of the matrix Schrödinger equation with a selfadjoint potential [7] and the matrix ZakharovShabat system $[2,3]$ when the geometric multiplicity may exceed one but the Jordan structure is trivial. The Schrödinger equation with energy dependent potential [5] provides an example of a treatment of dependency and norming constants when the geometric multiplicity is one but the algebraic multiplicity is arbitrary. The Jordan

Mathematics subject classification (2000): 34A55, 81U40.

Key words and phrases: Marchenko equation, norming constants, inverse scattering, matrix ZakharovShabat system. 
structure of eigenvalues of the (scalar) Zakharov-Shabat system has been analyzed by Tanaka [16], though with less detail than in the present article.

In this article we present a comprehensive treatment of norming constants and their appearance as parameters in the Marchenko integral kernels, where we base ourselves in part on results obtained in a recent $\mathrm{PhD}$ thesis $[11]$. We actually overcome two serious limitations of the existing treatments of this problem. One limitation, to cases where the geometric and algebraic multiplicities of the discrete eigenvalues coincide, has been discussed above. The other limitation concerns the construction, when the geometric and algebraic multiplicities equal one, of the norming constants from residues of analytic continuations of (matrix) functions involving a reflection coefficient $[2,3]$, which is only correct if the potential has compact support or has sufficient exponential decay for the required analytic continuations to exist. Such a construction of the norming constants is not an option for general potentials with $L^{1}$ entries. In the present work we present an alternative approach reminiscent of the construction prevailing in the case of the Schrödinger equation and sufficiently general to cover the case of general potentials with $L^{1}$ entries.

As in $[3,11]$, we deal with the matrix Zakharov-Shabat system, where the potential has $L^{1}$ entries but does not necessarily have any symmetry properties. At the same time we derive the symmetry properties of Marchenko integral kernels and norming constants valid in the focusing case, thus generalizing symmetry properties derived when the algebraic and geometric multiplicities coincide $[3,11]$. We avoid introducing any dependency constants (as done, e.g., in $[3,11]$ ). The appearance of matrices in the matrix Zakharov-Shabat system leads to the additional complication of having to study the dual matrix Zakharov-Shabat system along with the matrix Zakharov-Shabat system itself. This complication does not occur when dealing with the (scalar) Zakharov-Shabat system. Contrary to [11] where the dual matrix Zakharov-Shabat system was introduced as an afterthought in Appendix B, we now study the dual system alongside the matrix Zakharov-Shabat system from the start.

Let us discuss the contents of the various sections. In Section 2 we define the Jost function matrices and transition matrices and present their analyticity properties. In Section 3 the Jordan structure of the discrete eigenvalues is studied in detail. The reflection and transmission coefficients are introduced in Section 4 and their Fourier transform properties are given. We then go on to derive the Marchenko integral equations in Section 5, first if there are no discrete eigenvalues, then if the geometric and algebraic multiplicities of the eigenvalues coincide, and finally in general. Here we follow the outline of [11], where an analogous sequential presentation of Marchenko integral equations occurs. In general, eight seemingly different Marchenko integral kernels appear. In Section 6 we reduce their number to four, which is rather trivial in the absence of bound states or in the (scalar) Zakharov-Shabat case but requires extensive analysis in the general matrix Zakharov-Shabat case. This reduction does not require any symmetry assumptions on the potentials. In Section 7 we reduce their number to two in the focusing case by deriving some symmetry relations on taking adjoints.

Throughout we drop the notational system of $[6,11,17]$. Instead, we compromise between using Jost solutions as column vectors (as in $[2,3,15,18]$ ) and Jost solutions as square matrices (as in $[6,11,17]$ ), since either notation is advantageous in different 
circumstances. We distinguish between different related entities by using overlines for those functions analytic on the upper half complex plane. Wedges are used for entities involving the dual matrix Zakharov-Shabat system and tildes for entities associated with the eigenvalues in the lower half-plane. Compared to [3], we have changed the sign of the spectral parameter from $k$ to $\lambda=-k$.

By $\mathbb{C}^{+}$and $\mathbb{C}^{-}$we denote the open upper and lower complex half-planes, while $\overline{\mathbb{C}^{+}}$and $\overline{\mathbb{C}^{-}}$denote the closed upper and lower complex half-planes.

\section{Jost Matrices}

We consider the matrix Zakharov-Shabat system

$$
-i J X^{\prime}(\lambda, x)-V(x) X(\lambda, x)=\lambda X(\lambda, x), \quad x \in \mathbb{R},
$$

where prime denotes the $x$-derivative, $\lambda$ is the complex-valued spectral parameter, the solution $X(\lambda, x)$ may be either a column vector of length $n+m$ or a square matrix of order $n+m$, and

$$
J=\left[\begin{array}{cc}
I_{n} & 0_{n \times m} \\
0_{m \times n} & -I_{m}
\end{array}\right], \quad V(x)=i\left[\begin{array}{cc}
0_{n \times n} & q(x) \\
r(x) & 0_{m \times m}
\end{array}\right] .
$$

Here $I_{p}$ stands for the identity matrix of order $p$ and $0_{p_{1} \times p_{2}}$ for the $p_{1} \times p_{2}$ matrix with zero entries. Further, the potentials $q(x)$ and $r(x)$ are complex $n \times m$ and $m \times n$ matrices with entries in $L^{1}(\mathbb{R})$. Writing a dagger for the conjugate transpose of a matrix, we have $r(x)=q(x)^{\dagger}$ in the focusing case and $r(x)=-q(x)^{\dagger}$ in the defocusing case. To subsequently derive symmetry relations we also deal with the dual matrix Zakharov-Shabat system

$$
i Y^{\prime}(\lambda, x) J+Y(\lambda, x) V(x)=\lambda Y(\lambda, x),
$$

where $Y(\lambda, x)$ is a row vector of length $n+m$ or a square matrix of order $n+m$ and $J$ and $V(x)$ are given by (2.2). Here we note the sign change in the left-hand side when passing from (2.1) to (2.3).

By the Jost matrix from the left $\Psi(\lambda, x)$ and the Jost matrix from the right $\Phi(\lambda, x)$ we mean the unique $(n+m) \times(n+m)$ matrix solutions of $(2.1)$ satisfying the asymptotic conditions

$$
\begin{aligned}
\Psi(\lambda, x)=[\bar{\psi}(\lambda, x) \quad \psi(\lambda, x)] & =\left[\begin{array}{cc}
e^{i \lambda x} I_{n} & 0_{n \times m} \\
0_{m \times n} & e^{-i \lambda x} I_{m}
\end{array}\right]+o(1) \\
& =e^{i \lambda J x}+o(1), \quad x \rightarrow+\infty \\
\Phi(\lambda, x)=[\phi(\lambda, x) \quad \bar{\phi}(\lambda, x)] & =\left[\begin{array}{cc}
e^{i \lambda x} I_{n} & 0_{n \times m} \\
0_{m \times n} & e^{-i \lambda x} I_{m}
\end{array}\right]+o(1) \\
& =e^{i \lambda J x}+o(1), \quad x \rightarrow-\infty
\end{aligned}
$$

where $\lambda \in \mathbb{R}$ and $\bar{\psi}(\lambda, x), \psi(\lambda, x), \phi(\lambda, x)$, and $\bar{\phi}(\lambda, x)$ are the submatrices with $n+m$ rows and $n, m, n$, and $m$ columns, respectively, which are usually called 
Jost solutions $[1,2,3,4,18]$. Observing that $(2.1)$ implies $(\partial / \partial x) \operatorname{det} X(\lambda, x)=$ $i \lambda(n-m) \operatorname{det} X(\lambda, x)$ (cf. [9, Theorem I 7.3]), we get

$$
\operatorname{det} \Psi(\lambda, x)=\operatorname{det} \Phi(\lambda, x)=e^{i \lambda(n-m) x}, \quad \lambda \in \mathbb{R},
$$

which guarantees the nonsingularity of the Jost matrices for $\lambda \in \mathbb{R}$. Using the identities

$$
\begin{aligned}
J V(x) & =-V(x) J, \\
\frac{\partial}{\partial x} X(\lambda, x)^{-1} & =-X(\lambda, x)^{-1} X^{\prime}(\lambda, x) X(\lambda, x)^{-1},
\end{aligned}
$$

we easily see that the inverses $\Psi(\lambda, x)^{-1}$ and $\Phi(\lambda, x)^{-1}$ of the Jost matrices are the unique solutions of the dual matrix Zakharov-Shabat system (2.3) satisfying

$$
\begin{aligned}
& \check{\Psi}(\lambda, x)=\left[\begin{array}{l}
\check{\psi}(\lambda, x) \\
\check{\psi}(\lambda, x)
\end{array}\right] \stackrel{\text { def }}{=} \Psi(\lambda, x)^{-1}=e^{-i \lambda J x}+o(1), \quad x \rightarrow+\infty, \\
& \check{\Phi}(\lambda, x)=\left[\begin{array}{l}
\check{\phi}(\lambda, x) \\
\check{\phi}(\lambda, x)
\end{array}\right] \stackrel{\text { def }}{=} \Phi(\lambda, x)^{-1}=e^{-i \lambda J x}+o(1), \quad x \rightarrow-\infty .
\end{aligned}
$$

where $\check{\psi}(\lambda, x)$ and $\check{\phi}(\lambda, x)$ are $n \times(n+m)$ matrices and $\check{\psi}(\lambda, x)$ and $\check{\phi}(\lambda, x)$ are $m \times(n+m)$ matrices called the dual Jost solutions. The matrices $\check{\Psi}(\lambda, x)$ and $\check{\Phi}(\lambda, x)$ are called the dual Jost matrices.

Since (2.1) is a first order system and (2.5) is true, there exist, for $\lambda \in \mathbb{R}$, square matrices $A_{l}(\lambda)$ and $A_{r}(\lambda)$ of order $n+m$ such that

$$
\Psi(\lambda, x)=\Phi(\lambda, x) A_{l}(\lambda), \quad \Phi(\lambda, x)=\Psi(\lambda, x) A_{r}(\lambda) .
$$

These so-called transition matrices $A_{l}(\lambda)$ and $A_{r}(\lambda)$ are each others inverses and have unit determinant. Moreover, we have the asymptotic conditions

$$
\begin{aligned}
& \Psi(\lambda, x)=[\bar{\psi}(\lambda, x) \quad \psi(\lambda, x)]=e^{i \lambda J x} A_{l}(\lambda)+o(1), \quad x \rightarrow-\infty, \\
& \Phi(\lambda, x)=[\phi(\lambda, x) \quad \bar{\phi}(\lambda, x)]=e^{i \lambda J x} A_{r}(\lambda)+o(1), \quad x \rightarrow+\infty .
\end{aligned}
$$

Analogously, we derive from (2.8) the asymptotic conditions

$$
\begin{aligned}
& \check{\Psi}(\lambda, x)=\left[\begin{array}{l}
\check{\psi}(\lambda, x) \\
\check{\psi}(\lambda, x)
\end{array}\right]=\Psi(\lambda, x)^{-1}=A_{r}(\lambda) e^{-i \lambda J x}+o(1), \quad x \rightarrow-\infty, \\
& \check{\Phi}(\lambda, x)=\left[\begin{array}{l}
\check{\phi}(\lambda, x) \\
\check{\phi}(\lambda, x)
\end{array}\right]=\Phi(\lambda, x)^{-1}=A_{l}(\lambda) e^{-i \lambda J x}+o(1), \quad x \rightarrow+\infty,
\end{aligned}
$$

where we have used that $A_{l}(\lambda)^{-1}=A_{r}(\lambda)$. 
Proposition 2.1. Let $X(\lambda, x)$ and $Y(\lambda, x)$ be solutions of (2.1) and (2.3), respectively. Then for each $\lambda \in \mathbb{C}$ we have

$$
\frac{\partial}{\partial x}(Y(\lambda, x) X(\lambda, x))=0
$$

Proof. Using (2.5) and (2.6) we compute

$$
\begin{aligned}
\frac{\partial}{\partial x}(Y(\lambda, x) X(\lambda, x)) & =Y(\lambda, x) \frac{\partial X}{\partial x}(\lambda, x)+\frac{\partial Y}{\partial x}(\lambda, x) X(\lambda, x) \\
& =Y(\lambda, x)[i J(\lambda+V(x)) X(\lambda, x)]+[-i Y(\lambda, x)(\lambda-V(x)) J] X(\lambda, x) \\
& =i Y(\lambda, x)[J V(x)+V(x) J] X(\lambda, x)=0,
\end{aligned}
$$

as claimed.

In the focusing case $\left(V(x)^{\dagger}=-V(x)\right)$ any solution $X(\lambda, x)$ of $(2.1)$ induces a solution $Y(\lambda, x)=X(\bar{\lambda}, x)^{\dagger}$ of (2.3). In the defocusing case $\left(V(x)^{\dagger}=V(x)\right)$ any solution $X(\lambda, x)$ of $(2.1)$ induces a solution $Y(\lambda, x)=J X(\bar{\lambda}, x)^{\dagger} J$ of (2.3). Further, $\left[e^{-i \bar{\lambda} J x}\right]^{\dagger}=e^{-i \lambda J x}$ commutes with $J$ and $J^{2}=I_{n+m}$. As a result, we have for $\lambda \in \mathbb{R}$

$$
\begin{cases}\Psi(\lambda, x)^{-1}=\Psi(\lambda, x)^{\dagger} \text { and } \Phi(\lambda, x)^{-1}=\Phi(\lambda, x)^{\dagger}, & \text { focusing case, } \\ \Psi(\lambda, x)^{-1}=J \Psi(\lambda, x)^{\dagger} J \quad \text { and } \Phi(\lambda, x)^{-1}=J \Phi(\lambda, x)^{\dagger} J, & \text { defocusing case. }\end{cases}
$$

Thus in the focusing case we have for $\lambda \in \mathbb{R}$

$$
\begin{cases}\bar{\psi}(\lambda, x)^{\dagger}=\check{\psi}(\lambda, x), & \psi(\lambda, x)^{\dagger}=\overline{\check{\psi}}(\lambda, x), \\ \bar{\phi}(\lambda, x)^{\dagger}=\check{\phi}(\lambda, x), & \phi(\lambda, x)^{\dagger}=\bar{\phi}(\lambda, x) .\end{cases}
$$

Moreover, we have for $\lambda \in \mathbb{R}$

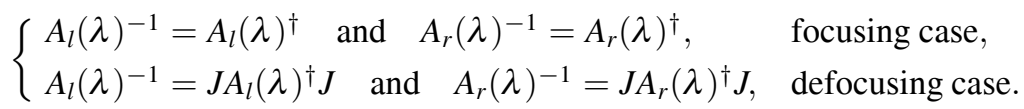

In other words, the Jost matrices and the transition matrices are unitary in the focusing case and $J$-unitary in the defocusing case.

Writing (2.1) in the form

$$
\frac{\partial}{\partial y}\left(e^{i \lambda J(x-y)} X(\lambda, y)\right)=i J e^{i \lambda J(x-y)} V(y) X(\lambda, y)
$$

and integrating with respect to $y$ we derive the Volterra integral equations

$$
\begin{aligned}
& \Psi(\lambda, x)=e^{i \lambda J x}-i J \int_{x}^{\infty} d y e^{i \lambda J(x-y)} V(y) \Psi(\lambda, y), \\
& \Phi(\lambda, x)=e^{i \lambda J x}-i J \int_{-\infty}^{x} d y e^{i \lambda J(x-y)} V(y) \Phi(\lambda, x) .
\end{aligned}
$$

For $\lambda \in \mathbb{R}$ Eqs. (2.11) imply the existence of unique solutions of (2.1) which satisfy (2.4), and hence justify introducing the Jost matrices a posteriori. Analogously, writing (2.3) in the form

$$
\frac{\partial}{\partial x}\left(Y(\lambda, y) e^{-i \lambda J(x-y)}\right)=i Y(\lambda, y) V(y) e^{-i \lambda J(x-y)} J
$$


and integrating with respect to $y$ we derive the alternative Volterra integral equations

$$
\begin{aligned}
& \Psi(\lambda, x)^{-1}=e^{-i \lambda J x}-i \int_{x}^{\infty} d y \Psi(\lambda, y)^{-1} V(y) e^{-i \lambda J(x-y)} J, \\
& \Phi(\lambda, x)^{-1}=e^{-i \lambda J x}+i \int_{-\infty}^{x} d y \Phi(\lambda, y)^{-1} V(y) e^{-i \lambda J(x-y)} J .
\end{aligned}
$$

For $\lambda \in \mathbb{R}$ Eqs. (2.12) imply the existence of unique solutions of (2.3) which satisfy (2.7).

The following proposition can be proved as its counterpart in $[6,17,11]$. Contrary to the practice in $[6,17,11]$, it is stated in terms of Jost functions instead of Faddeev matrices.

PROPOSITION 2.2. Suppose the entries of $q(x)$ and $r(x)$ belong to $L^{1}(\mathbb{R})$. Then the following statements are true:

(i) For each $x \in \mathbb{R}, e^{-i \lambda x} \bar{\psi}(\lambda, x), e^{i \lambda x} \bar{\phi}(\lambda, x), e^{i \lambda x} \bar{\psi}(\lambda, x)$, and $e^{-i \lambda x} \bar{\varnothing}(\lambda, x)$ can be extended to matrix functions that are continuous in $\lambda \in \overline{\mathbb{C}^{+}}$and analytic in $\lambda \in \mathbb{C}^{+}$. Moreover, these functions tend to

$$
\left[\begin{array}{c}
I_{n} \\
0_{m \times n}
\end{array}\right], \quad\left[\begin{array}{c}
0_{n \times m} \\
I_{m}
\end{array}\right], \quad\left[\begin{array}{ll}
0_{m \times n} & I_{m}
\end{array}\right], \quad\left[\begin{array}{ll}
I_{n} & 0_{n \times m}
\end{array}\right],
$$

respectively, as $|\lambda| \rightarrow \infty$ within $\overline{\mathbb{C}^{+}}$.

(ii) For each $x \in \mathbb{R}, e^{i \lambda x} \psi(\lambda, x), e^{-i \lambda x} \phi(\lambda, x), e^{i \lambda x} \check{\psi}(\lambda, x)$, and $e^{-i \lambda x} \check{\phi}(\lambda, x)$ can be extended to matrix functions that are continuous in $\lambda \in \overline{\mathbb{C}^{-}}$and analytic in $\lambda \in \mathbb{C}^{-}$. Moreover, these functions tend to

$$
\left[\begin{array}{c}
0_{n \times m} \\
I_{m}
\end{array}\right], \quad\left[\begin{array}{c}
I_{n} \\
0_{m \times n}
\end{array}\right], \quad\left[\begin{array}{ll}
I_{n} & 0_{n \times m}
\end{array}\right], \quad\left[\begin{array}{ll}
0_{m \times n} & I_{m}
\end{array}\right]
$$

respectively, as $|\lambda| \rightarrow \infty$ within $\overline{\mathbb{C}^{-}}$.

Before stating the next proposition (cf. $[6,17,11]$ ) we introduce a partitioning of square matrices of order $n+m$ into four submatrices which have the subscripts $1,2,3,4$ :

$$
Z=\left[\begin{array}{ll}
Z_{1} & Z_{2} \\
Z_{3} & Z_{4}
\end{array}\right]
$$

where $Z_{1}$ is $n \times n, Z_{2}$ is $n \times m, Z_{3}$ is $m \times n$, and $Z_{4}$ is $m \times m$.

PROPOSITION 2.3. Suppose the entries of $q(x)$ and $r(x)$ belong to $L^{1}(\mathbb{R})$. Then the following statements are true:

(i) The matrix functions $A_{l 1}(\lambda)$ and $A_{r 4}(\lambda)$ are continuous in $\lambda \in \overline{\mathbb{C}^{+}}$, are analytic in $\lambda \in \mathbb{C}^{+}$, and tend to the identity matrix as $|\lambda| \rightarrow \infty$ in $\overline{\mathbb{C}^{+}}$.

(ii) The matrix functions $A_{r 1}(\lambda)$ and $A_{l 4}(\lambda)$ are continuous in $\lambda \in \overline{\mathbb{C}^{-}}$, are analytic in $\lambda \in \mathbb{C}^{-}$, and tend to the identity matrix as $|\lambda| \rightarrow \infty$ in $\overline{\mathbb{C}^{-}}$.

(iii) The matrix functions $A_{l 2}(\lambda), A_{r 3}(\lambda), A_{l 3}(\lambda)$, and $A_{r 2}(\lambda)$ are continuous in $\lambda \in \mathbb{R}$ and vanish as $\lambda \rightarrow \pm \infty$. 


\section{Jordan Chains of the Hamiltonian}

In this section we prove that for $\lambda \in \mathbb{C}^{+}$the matrix Zakharov-Shabat system (2.1) and the matrix functions $A_{l 1}(\lambda)$ and $A_{r 4}(\lambda)$ have the same eigenvalues, the same geometric and algebraic multiplicities, and hence the same Jordan structure. A similar statement is proved for $\lambda \in \mathbb{C}^{-}$.

a. Jordan structure of matrix and operator functions. Let $\Omega$ be an open subset of the complex plane, $\mathscr{X}$ and $\mathscr{Y}$ complex Banach spaces, and $\mathscr{L}(\mathscr{X}, \mathscr{Y})$ the Banach space of all bounded linear operators from $\mathscr{X}$ into $\mathscr{Y}$. Then $F$ is called analytic if the scalar function $\left\langle F(\cdot) x, y^{*}\right\rangle$ is analytic for any vector $x \in \mathscr{X}$ and any bounded linear functional $y^{*}$ on $\mathscr{Y}$. For matrix functions (i.e., for $\mathscr{X}=\mathbb{C}^{p_{1}}$ and $\mathscr{Y}=\mathbb{C}^{p_{2}}$ ) this is equivalent to the analyticity of each matrix entry of $F$. Given $\lambda_{0} \in \Omega$, we denote by $\mathscr{H}\left(\mathscr{L}(\mathscr{X}, \mathscr{Y}), \lambda_{0}\right)$ the set of all germs of $\mathscr{L}(\mathscr{X})$-valued analytic functions in a neighborhood of $\lambda_{0}$, i.e., identifying analytic functions when they coincide on some neighborhood of $\lambda_{0}$. Given $F \in \mathscr{H}\left(\mathscr{L}(\mathscr{X}, \mathscr{Y}), \lambda_{0}\right)$ and writing

$$
F(\lambda)=\sum_{j=0}^{\infty}\left(\lambda-\lambda_{0}\right)^{j} F_{j}, \quad\left|\lambda-\lambda_{0}\right| \leqslant \varepsilon,
$$

we define the linear operator $\Xi_{s}\left(F ; \lambda_{0}\right)$ from $\mathscr{X}^{s}$ into $\mathscr{Y}^{s}$ by

$$
\Xi_{s}\left(F ; \lambda_{0}\right)=\left[\begin{array}{ccccc}
F_{0} & 0 & \cdots & \cdots & \cdots \\
F_{1} & F_{0} & 0 & \cdots & \cdots \\
\vdots & & \ddots & 0 & \\
\vdots & & & \ddots & \\
F_{s-1} & F_{s-2} & \cdots & \cdots & F_{0}
\end{array}\right],
$$

where $s=1,2, \ldots$ and $\mathscr{X}^{s}$ and $\mathscr{Y}^{s}$ are the direct sums of $s$ copies of $\mathscr{X}$ and $\mathscr{Y}$. Obviously, if $F \in \mathscr{H}\left(\mathscr{L}(\mathscr{Y}, \mathscr{Z}), \lambda_{0}\right), G \in \mathscr{H}\left(\mathscr{L}(\mathscr{X}, \mathscr{Y}), \lambda_{0}\right)$, and $H \in$ $\mathscr{H}\left(\mathscr{L}(\mathscr{X}, \mathscr{Z}), \lambda_{0}\right)$, then

$$
\Xi_{s}\left(F ; \lambda_{0}\right) \Xi_{s}\left(G ; \lambda_{0}\right)=\Xi_{s}\left(H ; \lambda_{0}\right), \quad s=1,2, \ldots,
$$

whenever $F(\lambda) G(\lambda)=H(\lambda)$ in some neighborhood of $\lambda_{0}$.

If $F \in \mathscr{H}\left(\mathscr{L}(\mathscr{X}, \mathscr{Y}), \lambda_{0}\right)$ has invertible values in a deleted neighborhood of $\lambda_{0}$ and $F\left(\lambda_{0}\right)$ is a Fredholm operator, then the numbers

$$
\alpha_{s}\left(F, \lambda_{0}\right)=\operatorname{dim} \operatorname{Ker} \Xi_{s+1}\left(F ; \lambda_{0}\right)
$$

are nonnegative integers and for some positive integer $Q$ we have

$$
\begin{aligned}
\alpha_{0}\left(F, \lambda_{0}\right) & \leqslant \alpha_{1}\left(F, \lambda_{0}\right) \leqslant \cdots \leqslant \alpha_{Q-1}\left(F, \lambda_{0}\right) \\
& <\alpha_{Q}\left(F, \lambda_{0}\right)=\alpha_{Q+1}\left(F, \lambda_{0}\right)=\ldots<+\infty .
\end{aligned}
$$

Then $Q$ is the order of the pole $F(\cdot)^{-1}$ in $\lambda_{0}$ and the integers $\left\{\alpha_{s}\left(F, \lambda_{0}\right)\right\}_{s=0}^{\infty}$ are called the Jordan characteristics of $F$ in $\lambda_{0}$. We then call $\alpha_{Q}\left(F, \lambda_{0}\right)$ the algebraic 
multiplicity and $\alpha_{0}\left(F, \lambda_{0}\right)$ the geometric multiplicity of $F$ at $\lambda_{0}$. A vector function

$$
x(\lambda)=\sum_{j=0}^{\infty}\left(\lambda-\lambda_{0}\right)^{j} x_{j}
$$

with coefficients $x_{j} \in \mathscr{X}$ and positive radius of convergence is called a root function of $F$ of order $s$ in $\lambda_{0}$ if $x_{0} \neq 0$ and

$$
F(\lambda) x(\lambda)=O\left(\left(\lambda-\lambda_{0}\right)^{s}\right), \quad \lambda \rightarrow \lambda_{0} .
$$

Then it is easily verified that

$$
\Xi_{s}\left(F ; \lambda_{0}\right)\left[\begin{array}{c}
x_{0} \\
x_{1} \\
\vdots \\
x_{s-1}
\end{array}\right]=\left[\begin{array}{c}
0 \\
0 \\
\vdots \\
0
\end{array}\right],
$$

where $s=1,2, \ldots$. Thus the root functions of $F$ of order $s$ in $\lambda_{0}$ correspond to the nontrivial elements of the null space of $\Xi_{s}\left(F ; \lambda_{0}\right)$ for any $s \geqslant 1$. Further, $\alpha_{s}\left(F, \lambda_{0}\right)$ coincides with the number of linearly independent root functions of $F$ in $\lambda_{0}$ that are polynomials of degree at most $s-1$. By a Jordan chain of $F$ in $\lambda_{0}$ of length $s$ we mean vectors $x_{0}, x_{1}, \ldots, x_{s-1}$ such that $x_{0} \neq 0$ and (3.1) holds.

At this point we compare the present definition of Jordan chain with that valid for $p \times p$ matrices $A$. Taking $\mathscr{X}=\mathscr{Y}=\mathbb{C}^{p}$ we define

$$
F(\lambda)=\lambda I_{p}-A \text {. }
$$

Then

$$
\Xi_{s}\left(F ; \lambda_{0}\right)=\left[\begin{array}{ccccc}
\lambda_{0} I_{p}-A & 0_{p \times p} & \ldots & \ldots & \ldots \\
I_{p} & \lambda_{0} I_{p}-A & 0 & \ldots & \ldots \\
\vdots & & \ddots & 0 & \\
\vdots & & & \ddots & \\
0 & 0 & \cdots & I_{p} & \lambda_{0} I_{p}-A
\end{array}\right]
$$

and

$$
\alpha_{s}\left(F, \lambda_{0}\right)=\operatorname{dim} \operatorname{Ker}\left(\lambda_{0} I_{p}-A\right)^{s+1},
$$

where $s=0,1,2, \ldots$. The number $Q$ is the length of the longest Jordan chain of $A$ corresponding to the eigenvalue $\lambda_{0}$. Thus we have indeed generalized the classical definition of Jordan chain to analytic operator-valued functions.

We now consider the effect of complex conjugation on Jordan structure. Let

$$
F(\lambda)=\sum_{j=0}^{\infty}\left(\lambda-\lambda_{0}\right)^{j} F_{j}
$$

be a $p \times p$ matrix function that is analytic in a neighborhood of $\lambda_{0}$, and let $\operatorname{det} F(\lambda) \neq 0$ in a deleted neighborhood of $\lambda_{0}$. Then it is easily verified that the $p \times p$ matrix function $F^{\dagger}$ defined by

$$
F^{\dagger}(\bar{\lambda})=\sum_{j=0}^{\infty}\left(\lambda-\overline{\lambda_{0}}\right)^{j}\left(F_{j}\right)^{\dagger}
$$


has the property that for $s=1,2,3, \ldots$

$$
\Xi_{s}\left(F^{\dagger} ; \overline{\lambda_{0}}\right)=\mathscr{S}_{s, p}\left[\Xi_{s}\left(F ; \lambda_{0}\right)\right]^{\dagger} \mathscr{S}_{s, p},
$$

where

$$
\mathscr{S}_{s, p}=\left[\delta_{j+k, s+1} I_{p}\right]_{j, k=1}^{s} .
$$

In fact, taking the conjugate transpose of $\Xi_{s}\left(F ; \lambda_{0}\right)$ amounts to taking the conjugate transpose of each $p \times p$ block matrix entry and reversing the order of $p \times p$ block rows and $p \times p$ columns, as a result of the $p \times p$ block Toeplitz structure of $\Xi_{s}\left(F ; \lambda_{0}\right)$.

b. Jordan structure of the Hamiltonian. We now apply the above definitions to the matrix Zakharov-Shabat system. It can be shown (e.g. [11]) that the Hamiltonian

$$
H=-i J \frac{d}{d x}-V(x)
$$

is closed and densely defined on the orthogonal direct sum of $n+m$ copies of $L^{2}(\mathbb{R})$ if the potentials $q(x)$ and $r(x)$ have their entries in $L^{1}(\mathbb{R})$. When these entries belong to $L^{1}(\mathbb{R}) \cap L^{2}(\mathbb{R})$, the domain of $H$ coincides with that of the free Hamiltonian

$$
H_{0}=-i J \frac{d}{d x} .
$$

The Hamiltonian $H$ is $J$-selfadjoint (i.e., $J H$ is selfadjoint) in the focusing case and selfadjoint in the defocusing case. Moreover, the spectrum of $H$ consists of the whole real line plus an at most countably infinite set of eigenvalues of finite algebraic multiplicity which can only accumulate on the real line or at infinity. In the defocusing case the spectrum of $H$ consists of the real line only, whereas in the focusing case it is merely symmetric upon reflection with respect to the real line.

Prior to determining the Jordan structure of the Hamiltonian $H$ at its nonreal eigenvalues, we observe that repeated differentiation of (2.1) with respect to $\lambda$ leads to the system of differential equations

$$
\begin{aligned}
& -i J X^{\prime}(\lambda, x)-V(x) X(\lambda, x)=\lambda X(\lambda, x), \\
& -i J\left(\frac{\partial X}{\partial \lambda}\right)^{\prime}(\lambda, x)-V(x) \frac{\partial X}{\partial \lambda}(\lambda, x)=\lambda \frac{\partial X}{\partial \lambda}(\lambda, x)+X(\lambda, x), \\
& -i J\left(\frac{1}{2 !} \frac{\partial^{2} X}{\partial \lambda^{2}}\right)^{\prime}(\lambda, x)-V(x) \frac{1}{2 !} \frac{\partial^{2} X}{\partial \lambda^{2}}(\lambda, x)=\lambda \frac{1}{2 !} \frac{\partial^{2} X}{\partial \lambda^{2}}(\lambda, x)+\frac{\partial X}{\partial \lambda}(\lambda, x), \\
& -i J\left(\frac{1}{s !} \frac{\partial^{s} X}{\partial \lambda^{s}}\right)^{\prime}(\lambda, x)-V(x) \frac{1}{s !} \frac{\partial^{s} X}{\partial \lambda^{s}}(\lambda, x)=\lambda \frac{1}{s !} \frac{\partial^{s} X}{\partial \lambda^{s}}(\lambda, x)+\frac{1}{(s-1) !} \frac{\partial^{s-1} X}{\partial \lambda^{s-1}},
\end{aligned}
$$

where the prime denotes differentiation with respect to $x$.

For $n=m=1$, similar (though less detailed) results as in the following Theorem 3.1 have been obtained by Tanaka [16]. 
THEOREM 3.1. Let $\lambda_{0} \in \mathbb{C}^{+}$be an eigenvalue of $H$. Then the Hamiltonian $H$ and the matrix functions $A_{l 1}(\lambda)$ and $A_{r 4}(\lambda)$ have the same Jordan structure at $\lambda_{0}$ :

$$
\operatorname{dim} \operatorname{Ker} \Xi_{s}\left(\lambda-H ; \lambda_{0}\right)=\operatorname{dim} \operatorname{Ker} \Xi_{s}\left(A_{l 1} ; \lambda_{0}\right)=\operatorname{dim} \operatorname{Ker} \Xi_{s}\left(A_{r 4} ; \lambda_{0}\right),
$$

where $s=1,2, \ldots$ Instead, if $\lambda_{0} \in \mathbb{C}^{-}$is an eigenvalue of $H$, then the Hamiltonian $H$ and the matrix functions $A_{r 1}(\lambda)$ and $A_{l 4}(\lambda)$ have the same Jordan structure at $\lambda_{0}$ :

$$
\operatorname{dim} \operatorname{Ker} \Xi_{s}\left(\lambda-H ; \lambda_{0}\right)=\operatorname{dim} \operatorname{Ker} \Xi_{s}\left(A_{r 1} ; \lambda_{0}\right)=\operatorname{dim} \operatorname{Ker} \Xi_{s}\left(A_{l 4} ; \lambda_{0}\right),
$$

where $s=1,2, \ldots$

Proof. Every column vector solution of (2.1) for $\lambda=\lambda_{0} \in \mathbb{C}^{+}$has one of the following equivalent forms:

$$
u(x)=\bar{\psi}(\lambda, x) \varepsilon=\bar{\phi}(\lambda, x) \eta, \quad x \in \mathbb{R},
$$

where $0 \neq \varepsilon \in \mathbb{C}^{n}$ and $0 \neq \eta \in \mathbb{C}^{m}$. Then (2.4) and (2.8) imply

$$
u(x)= \begin{cases}e^{i \lambda_{0} J x}\left[\begin{array}{c}
\varepsilon \\
0_{m \times 1}
\end{array}\right]+o(1)=e^{i \lambda_{0} J x} A_{r}\left(\lambda_{0}\right)\left[\begin{array}{c}
0_{n \times 1} \\
\eta
\end{array}\right]+o(1), & x \rightarrow+\infty, \\
e^{i \lambda_{0} J x}\left[\begin{array}{c}
0_{n \times 1} \\
\eta
\end{array}\right]+o(1)=e^{i \lambda_{0} J x} A_{l}\left(\lambda_{0}\right)\left[\begin{array}{c}
\varepsilon \\
0_{m \times 1}
\end{array}\right]+o(1), & x \rightarrow-\infty .\end{cases}
$$

In order for the entries of $u(x)$ to belong to $L^{2}(\mathbb{R})$ it is necessary and sufficient that

$$
A_{l 1}\left(\lambda_{0}\right) \varepsilon=0_{n \times 1}, \quad A_{r 4}\left(\lambda_{0}\right) \eta=0_{m \times 1} .
$$

Thus the eigenvalues of (2.1) in $\mathbb{C}^{+}$are the zeros of $\operatorname{det} A_{l 1}(\lambda)$ or $\operatorname{det} A_{r 4}(\lambda)$.

Putting

$$
\Psi^{s}(\lambda, x)=\left[\bar{\psi}^{s}(\lambda, x) \quad \psi^{s}(\lambda, x)\right]=\frac{1}{s !} \frac{\partial^{s} \Psi}{\partial \lambda^{s}}(\lambda, x),
$$

we depart from $\varepsilon_{0} \in \mathbb{C}^{n}$ satisfying $A_{l 1}\left(\lambda_{0}\right) \varepsilon_{0}=0$ with $\lambda_{0} \in \mathbb{C}^{+}$and define $\varepsilon_{1}, \ldots, \varepsilon_{s-1}$ to satisfy

$$
\begin{aligned}
& u_{0}(x)=\bar{\psi}^{0}\left(\lambda_{0}, x\right) \varepsilon_{0}, \\
& u_{1}(x)=\bar{\psi}^{1}\left(\lambda_{0}, x\right) \varepsilon_{0}+\bar{\psi}^{0}\left(\lambda_{0}, x\right) \varepsilon_{1}, \\
& u_{2}(x)=\bar{\psi}^{2}\left(\lambda_{0}, x\right) \varepsilon_{0}+\bar{\psi}^{1}\left(\lambda_{0}, x\right) \varepsilon_{1}+\bar{\psi}^{0}\left(\lambda_{0}, x\right) \varepsilon_{2} \text {, } \\
& u_{s-1}(x)=\sum_{\sigma=0}^{s-1} \bar{\psi}^{\sigma}\left(\lambda_{0}, x\right) \varepsilon_{s-\sigma-1} .
\end{aligned}
$$

Then $\left\{u_{0}, u_{1}, \ldots, u_{s-1}\right\}$ is a Jordan chain of length $s$ of $H$ at the eigenvalue $\lambda_{0}$ if and only if $0 \neq \varepsilon_{0} \in \mathbb{C}^{n}$ and $u_{0}(-\infty)=u_{1}(-\infty)=\ldots=u_{s-1}(-\infty)=0$. Computing 
the asymptotic behavior of each of the vectors $u_{0}(x), u_{1}(x), \ldots, u_{s-1}(x)$ we obtain for $t=1, \ldots, s-1$

$$
\left[\begin{array}{ll}
0_{m \times n} & I_{m}
\end{array}\right] u_{t}(x)= \begin{cases}e^{i \lambda_{0} x} \sum_{\sigma=0}^{t} \frac{(i x)^{\sigma}}{\sigma !} \varepsilon_{t-\sigma}+o(1), & x \rightarrow+\infty \\
\sum_{\sigma=0}^{t} \frac{1}{\sigma !}\left(\frac{d}{d \lambda_{0}}\right)^{\sigma} e^{i \lambda_{0} x} A_{l 1}\left(\lambda_{0}\right) \varepsilon_{t-\sigma}+o(1), & x \rightarrow-\infty .\end{cases}
$$

Writing the second line as

$$
e^{i \lambda_{0} x} \sum_{r=0}^{t} \frac{(i x)^{r}}{r !} \sum_{\rho=0}^{t-r} \frac{1}{\rho !}\left(\frac{d}{d \lambda_{0}}\right)^{\rho} A_{l 1}\left(\lambda_{0}\right) \varepsilon_{t-r-\rho},
$$

we obtain the identity

$$
\Xi_{s}\left(A_{l 1} ; \lambda_{0}\right)\left[\begin{array}{c}
\varepsilon_{0} \\
\vdots \\
\varepsilon_{s-1}
\end{array}\right]=0
$$

where $0 \neq \varepsilon_{0} \in \mathbb{C}^{n}$.

Analogously, putting

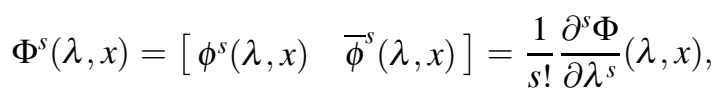

we depart from $\eta_{0} \in \mathbb{C}^{m}$ satisfying $A_{r 4}\left(\lambda_{0}\right) \eta_{0}=0$ with $\lambda_{0} \in \mathbb{C}^{+}$and define $\eta_{1}, \ldots, \eta_{s-1}$ to satisfy

$$
\begin{aligned}
& u_{0}(x)=\bar{\phi}^{0}\left(\lambda_{0}, x\right) \eta_{0} \\
& u_{1}(x)=\bar{\phi}^{1}\left(\lambda_{0}, x\right) \eta_{0}+\bar{\phi}^{0}\left(\lambda_{0}, x\right) \eta_{1} \text {, } \\
& u_{2}(x)=\bar{\phi}^{2}\left(\lambda_{0}, x\right) \eta_{0}+\bar{\phi}^{1}\left(\lambda_{0}, x\right) \eta_{1}+\bar{\phi}^{0}\left(\lambda_{0}, x\right) \eta_{2} \text {, } \\
& u_{s-1}(x)=\sum_{\sigma=0}^{s-1} \bar{\phi}^{\sigma}\left(\lambda_{0}, x\right) \eta_{s-\sigma-1}
\end{aligned}
$$

Then $\left\{u_{0}, u_{1}, \ldots, u_{s-1}\right\}$ is a Jordan chain of length $s$ of $H$ at the eigenvalue $\lambda_{0}$ if and only if $0 \neq \eta_{0} \in \mathbb{C}^{m}$ and $u_{0}(+\infty)=u_{1}(+\infty)=\ldots=u_{s-1}(+\infty)=0$. Computing the asymptotic behavior of each of the vectors $u_{0}(x), u_{1}(x), \ldots, u_{s-1}(x)$ we obtain for $t=1, \ldots, s-1$

$$
\left[\begin{array}{ll}
I_{n} & 0_{n \times m}
\end{array}\right] u_{t}(x)= \begin{cases}e^{-i \lambda_{0} x} \sum_{\sigma=0}^{t} \frac{(-i x)^{\sigma}}{\sigma !} \eta_{t-\sigma}+o(1), & x \rightarrow-\infty \\
\sum_{\sigma=0}^{t} \frac{1}{\sigma !}\left(\frac{d}{d \lambda_{0}}\right)^{\sigma} e^{-i \lambda_{0} x} A_{r 4}\left(\lambda_{0}\right) \eta_{t-\sigma}+o(1), & x \rightarrow+\infty\end{cases}
$$


Writing the second line as

$$
e^{-i \lambda_{0} x} \sum_{r=0}^{t} \frac{(-i x)^{r}}{r !} \sum_{\rho=0}^{t-r} \frac{1}{\rho !}\left(\frac{d}{d \lambda_{0}}\right)^{\rho} A_{r 4}\left(\lambda_{0}\right) \eta_{t-r-\rho},
$$

we obtain the identity

$$
\Xi_{s}\left(A_{r 4} ; \lambda_{0}\right)\left[\begin{array}{c}
\eta_{0} \\
\vdots \\
\eta_{s-1}
\end{array}\right]=0
$$

where $0 \neq \eta_{0} \in \mathbb{C}^{m}$.

We have derived the Jordan structure of the Hamiltonian $H$ for eigenvalues $\lambda_{0} \in$ $\mathbb{C}^{+}$in two different ways, leading to (3.6) and (3.8), where the corresponding Jordan chains can be written in the respective forms

$$
\left\{\sum_{\sigma=0}^{t-1} \bar{\psi}^{\sigma}\left(\lambda_{0}, x\right) \varepsilon_{t-\sigma-1}\right\}_{t=1}^{s}, \quad\left\{\sum_{\sigma=0}^{t-1} \bar{\phi}^{\sigma}\left(\lambda_{0}, x\right) \eta_{t-\sigma-1}\right\}_{t=1}^{s} .
$$

Since these two constructions are equivalent, we necessarily have

$$
\operatorname{dim} \operatorname{Ker} \Xi_{s}\left(A_{l 1} ; \lambda_{0}\right)=\operatorname{dim} \operatorname{Ker} \Xi_{s}\left(A_{r 4} ; \lambda_{0}\right), \quad s=1,2, \ldots,
$$

as claimed.

In the same way we can write the Jordan chains corresponding to an eigenvalue $\tilde{\lambda}_{0} \in \mathbb{C}^{-}$of $H$ in the form

$$
\left\{\sum_{\sigma=0}^{t-1} \phi^{\sigma}\left(\tilde{\lambda}_{0}, x\right) \tilde{\varepsilon}_{t-\sigma-1}\right\}_{t=1}^{s}, \quad\left\{\sum_{\sigma=0}^{t-1} \psi^{\sigma}\left(\tilde{\lambda}_{0}, x\right) \tilde{\eta}_{t-\sigma-1}\right\}_{t=1}^{s},
$$

where $0 \neq \tilde{\varepsilon}_{0} \in \operatorname{Ker} A_{r 1}\left(\tilde{\lambda}_{0}\right)$ and $0 \neq \tilde{\eta}_{0} \in \operatorname{Ker} A_{l 4}\left(\tilde{\lambda}_{0}\right)$. We thus derive

$$
\operatorname{dim} \operatorname{Ker} \Xi_{s}\left(A_{r 1} ; \tilde{\lambda}_{0}\right)=\operatorname{dim} \operatorname{Ker} \Xi_{s}\left(A_{l 4} ; \tilde{\lambda}_{0}\right), \quad s=1,2, \ldots,
$$

which completes the proof.

COROLlaRY 3.2. Let $\lambda_{0} \in \mathbb{C} \backslash \mathbb{R}$. Then the geometric multiplicity of $\lambda_{0}$ as an eigenvalue of the Hamiltonian $H$ does not exceed $\min (n, m)$.

Proof. The geometric multiplicity of $\lambda_{0} \in \mathbb{C}^{+}$as an eigenvalue of $H$ is equal to

$$
\operatorname{dim} \operatorname{Ker} A_{l 1}\left(\lambda_{0}\right)=\operatorname{dim} \operatorname{Ker} A_{r 4}\left(\lambda_{0}\right) .
$$

The left-hand side is at most $n$ and the right-hand side is at most $m$. The same argument applies to $\lambda_{0} \in \mathbb{C}^{-}$.

c. Jordan structure of the dual Hamiltonian. Instead of the eigenfunctions and generalized eigenfunctions of the matrix Zakharov-Shabat system (2.1), we now 
consider the Jordan structure of the dual matrix Zakharov-Shabat system (2.3). Repeated differentiation of (2.3) with respect to $\lambda$ leads to the system of differential equations

$$
\begin{aligned}
& i Y^{\prime}(\lambda, x) J+Y(\lambda, x) V(x)=\lambda Y(\lambda, x), \\
& i\left(\frac{\partial Y}{\partial \lambda}\right)^{\prime}(\lambda, x) J+\frac{\partial Y}{\partial \lambda}(\lambda, x) V(x)=\lambda \frac{\partial Y}{\partial \lambda}(\lambda, x)+Y(\lambda, x), \\
& i\left(\frac{1}{2 !} \frac{\partial^{2} Y}{\partial \lambda^{2}}\right)^{\prime}(\lambda, x) J+\frac{1}{2 !} \frac{\partial^{2} Y}{\partial \lambda^{2}}(\lambda, x) V(x)=\lambda \frac{1}{2 !} \frac{\partial^{2} Y}{\partial \lambda^{2}}(\lambda, x)+\frac{\partial Y}{\partial \lambda}(\lambda, x), \\
& i\left(\frac{1}{s !} \frac{\partial^{s} Y}{\partial \lambda^{s}}\right)^{\prime}(\lambda, x) J+\frac{1}{s !} \frac{\partial^{s} Y}{\partial \lambda^{s}}(\lambda, x) V(x)=\lambda \frac{1}{s !} \frac{\partial^{s} Y}{\partial \lambda^{s}}(\lambda, x)+\frac{1}{(s-1) !} \frac{\partial^{s-1} Y}{\partial \lambda^{s-1}},
\end{aligned}
$$

where the prime denotes differentiation with respect to $x$.

Every row vector solution of (2.3) for $\lambda=\lambda_{0} \in \mathbb{C}^{+}$has one of the following equivalent forms:

$$
v(x)=\theta \bar{\phi}\left(\lambda_{0}, x\right)=\zeta \overline{\breve{\psi}}\left(\lambda_{0}, x\right), \quad x \in \mathbb{R},
$$

where $0 \neq \theta \in \mathbb{C}^{1 \times n}$ and $0 \neq \zeta \in \mathbb{C}^{1 \times m}$ are complex row vectors. Then (2.7) and (2.9) imply

$$
v(x)= \begin{cases}{\left[\begin{array}{ll}
0_{1 \times n} & \zeta
\end{array}\right] e^{-i \lambda_{0} J x}+o(1)=\left[\begin{array}{ll}
\theta & 0_{1 \times m}
\end{array}\right] A_{l}\left(\lambda_{0}\right) e^{-i \lambda_{0} J x}+o(1),} & x \rightarrow+\infty \\
{\left[\begin{array}{ll}
\theta & 0_{1 \times m}
\end{array}\right] e^{-i \lambda_{0} J x}+o(1)=\left[\begin{array}{ll}
0_{1 \times n} & \zeta
\end{array}\right] A_{r}\left(\lambda_{0}\right) e^{-i \lambda_{0} J x}+o(1),} & x \rightarrow-\infty .\end{cases}
$$

In order for the entries of $v(x)$ to belong to $L^{2}(\mathbb{R})$ it is necessary and sufficient that

$$
\theta A_{l 1}\left(\lambda_{0}\right)=0_{1 \times n}, \quad \zeta A_{r 4}\left(\lambda_{0}\right)=0_{1 \times m} .
$$

Putting, in analogy with (3.5) and (3.7),

$$
\check{\Psi}^{s}(\lambda, x)=\left[\begin{array}{c}
\check{\psi}^{s}(\lambda, x) \\
\check{\psi}(\lambda, x)
\end{array}\right]=\frac{1}{s !} \frac{\partial^{s} \check{\Psi}}{\partial \lambda \lambda^{s}}(\lambda, x), \quad \check{\Phi}^{s}(\lambda, x)=\left[\begin{array}{l}
\check{\phi}(\lambda, x) \\
\check{\phi}(\lambda, x)
\end{array}\right]=\frac{1}{s !} \frac{\partial^{s} \check{\Phi}}{\partial \lambda s}(\lambda, x),
$$

we depart from $\theta_{0} \in \mathbb{C}^{1 \times n}$ and $\zeta \in \mathbb{C}^{1 \times m}$ such that $\theta_{0} A_{l 1}(\lambda)=0_{1 \times n}$ and $\zeta_{0} A_{r 4}(\lambda)=$ $0_{1 \times m}$, and define $\zeta_{1}, \ldots, \zeta_{s-1}$ and $\theta_{1}, \ldots, \theta_{s-1}$ to satisfy

$$
\begin{aligned}
& v_{0}(x)=\theta_{0} \overline{\check{\phi}}^{0}\left(\lambda_{0}, x\right)=\zeta_{0} \breve{\psi}^{0}\left(\lambda_{0}, x\right), \\
& v_{1}(x)=\theta_{0} \overline{\check{\phi}}^{1}\left(\lambda_{0}, x\right)+\theta_{1} \widetilde{\boldsymbol{\phi}}^{0}\left(\lambda_{0}, x\right)=\zeta_{0} \overline{\check{\psi}}^{1}\left(\lambda_{0}, x\right)+\zeta_{1} \breve{\psi}^{0}\left(\lambda_{0}, x\right) \text {, } \\
& v_{s-1}(x)=\sum_{\sigma=0}^{s-1} \theta_{s-\sigma-1} \overline{\check{\phi}}^{\sigma}\left(\lambda_{0}, x\right)=\sum_{\sigma=0}^{s-1} \zeta_{s-\sigma-1} \overline{\check{\psi}}^{\sigma}\left(\lambda_{0}, x\right) .
\end{aligned}
$$

Then $\left\{v_{0}, v_{1}, \ldots, v_{s-1}\right\}$ is a dual Jordan chain of length $s$ at the eigenvalue $\lambda_{0} \in \mathbb{C}^{+}$ if and only if $0 \neq \theta_{0} \in \mathbb{C}^{1 \times n}, 0 \neq \zeta_{0} \in \mathbb{C}^{1 \times m}$, and $v_{0}( \pm \infty)=v_{1}( \pm \infty)=\ldots=$ 
$v_{s-1}( \pm \infty)=0$. This is the case if and only if $0 \neq \theta_{0} \in \mathbb{C}^{1 \times n}, 0 \neq \zeta_{0} \in \mathbb{C}^{1 \times m}$, and for $t=0,1, \ldots, s-1$

$$
\sum_{\sigma=0}^{t} \theta_{t-\sigma} \frac{1}{\sigma !}\left(\frac{d}{d \lambda_{0}}\right)^{\sigma} e^{i \lambda_{0} x} A_{l 1}\left(\lambda_{0}\right)=\sum_{\sigma=0}^{t} \zeta_{t-\sigma} \frac{1}{\sigma !}\left(\frac{d}{d \lambda_{0}}\right)^{\sigma} e^{-i \lambda_{0} x} A_{r 4}\left(\lambda_{0}\right)=0 .
$$

The latter identity can be written as

$$
\begin{aligned}
e^{i \lambda_{0} x} \sum_{r=0}^{t} \frac{(i x)^{r}}{r !} \sum_{\rho=0}^{t-r} \theta_{t-r-\rho} \frac{1}{\rho !}\left(\frac{d}{d \lambda_{0}}\right)^{\rho} A_{l 1}\left(\lambda_{0}\right) & \\
& =e^{-i \lambda_{0} x} \sum_{r=0}^{t} \frac{(-i x)^{r}}{r !} \sum_{\rho=0}^{t-r} \zeta_{t-r-\rho} \frac{1}{\rho !}\left(\frac{d}{d \lambda_{0}}\right)^{\rho} A_{r 4}\left(\lambda_{0}\right)=0 .
\end{aligned}
$$

Consequently, $\left\{v_{0}, v_{1}, \ldots, v_{s-1}\right\}$ is a dual Jordan chain at the eigenvalue $\lambda \in \mathbb{C}^{+}$if and only if $0 \neq \theta_{0} \in \mathbb{C}^{1 \times n}, 0 \neq \zeta_{0} \in \mathbb{C}^{1 \times m}$, and

$$
\begin{aligned}
& {\left[\begin{array}{llll}
\theta_{s-1} & \theta_{s-2} & \ldots & \theta_{0}
\end{array}\right] \Xi_{s}\left(A_{l 1} ; \lambda_{0}\right)=0_{1 \times n s},} \\
& {\left[\begin{array}{llll}
\zeta_{s-1} & \zeta_{s-2} & \ldots & \zeta_{0}
\end{array}\right] \Xi_{s}\left(A_{r 4} ; \lambda_{0}\right)=0_{1 \times m s} .}
\end{aligned}
$$

We have therefore written the dual Jordan chains corresponding to the eigenvalue $\lambda_{0} \in \mathbb{C}^{+}$in either of the equivalent forms

$$
\left\{\sum_{\sigma=0}^{t-1} \theta_{t-\sigma-1} \overline{\check{\phi}}^{\sigma}\left(\lambda_{0}, x\right)\right\}_{t=1}^{s}, \quad\left\{\sum_{\sigma=0}^{t-1} \zeta_{t-\sigma-1} \overline{\check{\psi}}^{\sigma}\left(\lambda_{0}, x\right)\right\}_{t=1}^{s} .
$$

A similar result holds if $\lambda_{0}$ is an eigenvalue of $(2.3)$ in $\mathbb{C}^{-}$. Indeed, the corresponding Jordan chains have either of the equivalent forms

$$
\left\{\sum_{\sigma=0}^{t-1} \tilde{\theta}_{t-\sigma-1} \check{\psi}^{\sigma}\left(\lambda_{0}, x\right)\right\}_{t=1}^{s}, \quad\left\{\sum_{\sigma=0}^{t-1} \tilde{\zeta}_{t-\sigma-1} \check{\phi}^{\sigma}\left(\lambda_{0}, x\right)\right\}_{t=1}^{s},
$$

where

$$
\begin{aligned}
& {\left[\begin{array}{llll}
\tilde{\theta}_{s-1} & \tilde{\theta}_{s-2} & \ldots & \tilde{\theta}_{0}
\end{array}\right] \Xi_{s}\left(A_{r 1} ; \lambda_{0}\right)=0_{1 \times n s},} \\
& {\left[\begin{array}{llll}
\tilde{\zeta}_{s-1} & \tilde{\zeta}_{s-2} & \ldots & \tilde{\zeta}_{0}
\end{array}\right] \Xi_{s}\left(A_{l 4} ; \lambda_{0}\right)=0_{1 \times m s} .}
\end{aligned}
$$

\section{Reflection and Transmission Coefficients}

In this section we introduce the reflection and transmission coefficients and study their meromorphicity and symmetry properties.

a. Principal parts of transmission coefficients. Recalling that $A_{l 1}(\lambda)$ and $A_{r 4}(\lambda)$ are analytic in $\lambda \in \mathbb{C}^{+}$and approach the identity matrix as $|\lambda| \rightarrow+\infty$, we define the transmission coefficients

$$
T_{l}(\lambda)=A_{l 1}(\lambda)^{-1}, \quad T_{r}(\lambda)=A_{r 4}(\lambda)^{-1},
$$


as meromorphic matrix functions in $\lambda \in \mathbb{C}^{+}$. Similarly, we define

$$
\tilde{T}_{l}(\lambda)=A_{r 1}(\lambda)^{-1}, \quad \tilde{T}_{r}(\lambda)=A_{l 4}(\lambda)^{-1},
$$

as meromorphic matrix functions in $\lambda \in \mathbb{C}^{-}$. Then the poles of $T_{l}(\lambda)$ and $T_{r}(\lambda)$ in $\mathbb{C}^{+}$coincide with the eigenvalues $\lambda_{j}$ of $H$ in $\mathbb{C}^{+}$and the pole order $Q_{j}$ coincides with the length of the longest Jordan chain corresponding to the eigenvalue $\lambda_{j}$. We now write the principal parts of $T_{l}(\lambda)$ and $T_{r}(\lambda)$ at $\lambda_{j}$ as follows:

$$
\sum_{s=0}^{Q_{j}-1} \frac{i^{s+1} \tau_{l j s}}{\left(\lambda-\lambda_{j}\right)^{s+1}}, \quad \sum_{s=0}^{Q_{j}-1} \frac{i^{s+1} \tau_{r j s}}{\left(\lambda-\lambda_{j}\right)^{s+1}} .
$$

By the same token, the poles of $\tilde{T}_{l}(\lambda)$ and $\tilde{T}_{r}(\lambda)$ in $\mathbb{C}^{-}$coincide with the eigenvalues $\tilde{\lambda}_{j}$ of $H$ in $\mathbb{C}^{-}$and the pole order $\tilde{Q}_{j}$ coincides with the length of the longest Jordan chain corresponding to the eigenvalue $\tilde{\lambda}_{j}$. We write the principal parts of $\tilde{T}_{l}(\lambda)$ and $\tilde{T}_{r}(\lambda)$ at $\tilde{\lambda}_{j}$ as follows:

$$
\sum_{s=0}^{\tilde{Q}_{j}-1} \frac{(-i)^{s+1} \tilde{\tau}_{l j s}}{\left(\lambda-\tilde{\lambda}_{j}\right)^{s+1}}, \quad \sum_{s=0}^{\tilde{Q}_{j}-1} \frac{(-i)^{s+1} \tilde{\tau}_{r j s}}{\left(\lambda-\tilde{\lambda}_{j}\right)^{s+1}} .
$$

Let us now represent the transmission coefficients in the following form:

$$
\begin{aligned}
& T_{l}(\lambda)=T_{l}^{+}(\lambda)-\sum_{j} \sum_{s=0}^{Q_{j}-1} \int_{0}^{\infty} d \alpha \frac{\alpha^{s}}{s !} e^{-i \lambda \alpha} e^{i \lambda_{j} \alpha} \tau_{l j s}, \\
& T_{r}(\lambda)=T_{r}^{+}(\lambda)-\sum_{j} \sum_{s=0}^{Q_{j}-1} \int_{0}^{\infty} d \alpha \frac{\alpha^{s}}{s !} e^{-i \lambda \alpha} e^{i \lambda_{j} \alpha} \tau_{r j s}, \\
& \tilde{T}_{l}(\lambda)=\tilde{T}_{l}^{-}(\lambda)-\sum_{j} \sum_{s=0}^{\tilde{Q}_{j}-1} \int_{0}^{\infty} d \alpha \frac{\alpha^{s}}{s !} e^{i \lambda \alpha} e^{-i \tilde{\lambda}_{j} \alpha} \tilde{\tau}_{l j s}, \\
& \tilde{T}_{r}(\lambda)=\tilde{T}_{r}^{-}(\lambda)-\sum_{j} \sum_{s=0}^{\tilde{Q}_{j}-1} \int_{0}^{\infty} d \alpha \frac{\alpha^{s}}{s !} e^{i \lambda \alpha} e^{-i \tilde{\lambda}_{j} \alpha} \tilde{\tau}_{r j s},
\end{aligned}
$$

where $T_{l}^{+}(\lambda)$ and $T_{r}^{+}(\lambda)$ are continuous in $\lambda \in \overline{\mathbb{C}^{+}}$, are analytic in $\lambda \in \mathbb{C}^{+}$, and tend to the identity matrix as $\lambda \rightarrow \infty$ in $\overline{\mathbb{C}}^{+}$and $\tilde{T}_{l}^{-}(\lambda)$ and $\tilde{T}_{r}^{-}(\lambda)$ are continuous in $\lambda \in \overline{\mathbb{C}^{-}}$, are analytic in $\lambda \in \mathbb{C}^{-}$, and tend to the identity matrix as $\lambda \rightarrow \infty$ in $\overline{\mathbb{C}^{-}}$.

b. Modified Jost matrices and the scattering matrix. The first $n$ columns of the Jost matrices $\Psi(\lambda, x)$ and $\Phi(\lambda, x)$ have other analyticity properties than their last $m$ columns. This is also the case for the rows of $\check{\Psi}(\lambda, x)$ and $\check{\Phi}(\lambda, x)$. As in $[6,17,11]$, we therefore introduce modified Jost matrices by interchanging their columns in such a way that we obtain matrices that are analytic in $\lambda \in \mathbb{C}^{+}$and $\lambda \in \mathbb{C}^{-}$, respectively. 
We also define modified dual Jost matrices by suitable row interchange. More precisely, we define the modified Jost matrices and modified Jost functions by

$$
F_{+}(\lambda, x)=\left[\begin{array}{ll}
\bar{\psi}(\lambda, x) & \bar{\phi}(\lambda, x)
\end{array}\right], \quad F_{-}(\lambda, x)=\left[\begin{array}{ll}
\phi(\lambda, x) & \psi(\lambda, x)
\end{array}\right],
$$

and the modified dual Jost matrices and modified dual Jost functions by

$$
\check{F}_{+}(\lambda, x)=\left[\begin{array}{l}
\check{\phi}(\lambda, x) \\
\check{\psi}(\lambda, x)
\end{array}\right], \quad \check{F}_{-}(\lambda, x)=\left[\begin{array}{c}
\check{\psi}(\lambda, x) \\
\check{\phi}(\lambda, x)
\end{array}\right] .
$$

PROPOSITION 4.1. Suppose the entries of $q(x)$ and $r(x)$ belong to $L^{1}(\mathbb{R})$. Then for each $x \in \mathbb{R}, F_{ \pm}(\lambda, x) e^{-i \lambda J x}$ and $e^{i \lambda J x} \breve{F}_{ \pm}(\lambda, x)$ can be extended to matrix functions that are continuous in $\lambda \in \overline{\mathbb{C}^{ \pm}}$and analytic in $\lambda \in \mathbb{C}^{ \pm}$. Moreover, we have the asymptotic relations

$$
\begin{aligned}
& F_{+}(\lambda, x)=[\bar{\psi}(\lambda, x) \quad \bar{\phi}(\lambda, x)] \simeq \begin{cases}e^{i \lambda J x}\left[\begin{array}{cc}
I_{n} & A_{r 2}(\lambda) \\
0_{m \times n} & A_{r 4}(\lambda)
\end{array}\right], & x \rightarrow+\infty, \\
e^{i \lambda J x}\left[\begin{array}{cc}
A_{l 1}(\lambda) & 0_{n \times m} \\
A_{l 3} & (\lambda) I_{m}
\end{array}\right], & x \rightarrow-\infty,\end{cases} \\
& F_{-}(\lambda, x)=[\phi(\lambda, x) \quad \psi(\lambda, x)] \simeq \begin{cases}e^{i \lambda J x}\left[\begin{array}{cc}
A_{r 1}(\lambda) & 0_{n \times m} \\
A_{r 3}(\lambda) & I_{m}
\end{array}\right], & x \rightarrow+\infty, \\
e^{i \lambda J x}\left[\begin{array}{cc}
I_{n} & A_{l 2}(\lambda) \\
0_{m \times n} & A_{l 4}(\lambda)
\end{array}\right], & x \rightarrow-\infty,\end{cases} \\
& \breve{F}_{+}(\lambda, x)=[\check{\check{\phi}}(\lambda, x)] \simeq \begin{cases}{\left[\begin{array}{cc}
A_{l 1}(\lambda) & A_{l 2}(\lambda) \\
0_{m \times n} & I_{m}
\end{array}\right] e^{-i \lambda J x},} & x \rightarrow+\infty, \\
{\left[\begin{array}{cc}
I_{n} & 0_{n \times m} \\
A_{r 3}(\lambda) & A_{r 4}(\lambda)
\end{array}\right] e^{-i \lambda J x},} & x \rightarrow-\infty,\end{cases} \\
& \check{F}_{-}(\lambda, x)=\left[\begin{array}{c}
\breve{\psi}(\lambda, x) \\
\check{\phi}(\lambda, x)
\end{array}\right] \simeq\left\{\begin{array}{cc}
{\left[\begin{array}{cc}
I_{n} & 0_{n \times m} \\
A_{l 3}(\lambda) & A_{l 4}(\lambda)
\end{array}\right] e^{-i \lambda J x},} & x \rightarrow+\infty, \\
{\left[\begin{array}{cc}
A_{r 1}(\lambda) & A_{r 2}(\lambda) \\
0_{m \times n} & I_{m}
\end{array}\right] e^{-i \lambda J x},} & x \rightarrow-\infty .
\end{array}\right.
\end{aligned}
$$

In the remainder of this article we make the usual technical hypothesis that the matrices $A_{l 1}(\lambda), A_{l 4}(\lambda), A_{r 1}(\lambda)$, and $A_{r 4}(\lambda)$ are invertible for all $\lambda \in \mathbb{R}$. Under this hypothesis it can easily be shown using (4.6a), (4.6b), and $A_{l}(\lambda) A_{r}(\lambda)=$ $A_{r}(\lambda) A_{l}(\lambda)=I_{n+m}$ that

$$
F_{+}(\lambda, x)^{-1} F_{-}(\lambda, x) \rightarrow J S(\lambda) J, \quad x \rightarrow \pm \infty,
$$

where

is called the scattering matrix and

$$
S(\lambda)=\left[\begin{array}{ll}
T_{l}(\lambda) & R(\lambda) \\
L(\lambda) & T_{r}(\lambda)
\end{array}\right]
$$

$$
\begin{aligned}
& L(\lambda)=A_{l 3}(\lambda) A_{l 1}(\lambda)^{-1}=-A_{r 4}(\lambda)^{-1} A_{r 3}(\lambda), \\
& R(\lambda)=A_{r 2}(\lambda) A_{r 4}(\lambda)^{-1}=-A_{l 1}(\lambda)^{-1} A_{l 2}(\lambda),
\end{aligned}
$$


are called reflection coefficient from the right and reflection coefficient from the left, respectively. Since $F_{+}(\lambda, x) e^{-i \lambda J x}$ and $F_{-}(\lambda, x) e^{-i \lambda J x}$ satisfy the same first order linear system of differential equations, we obtain the Riemann-Hilbert problem

$$
F_{-}(\lambda, x)=F_{+}(\lambda, x) J S(\lambda) J .
$$

Analogously, we have

$$
F_{-}(\lambda, x)^{-1} F_{+}(\lambda, x) \rightarrow J \tilde{S}(\lambda) J, \quad x \rightarrow \pm \infty,
$$

where

$$
\tilde{S}(\lambda)=\left[\begin{array}{ll}
\tilde{T}_{l}(\lambda) & \tilde{L}_{(\lambda)} \\
\tilde{R}(\lambda) & \tilde{T}_{r}(\lambda)
\end{array}\right]
$$

is also called scattering matrix and

$$
\begin{aligned}
& \tilde{L}(\lambda)=A_{l 2}(\lambda) A_{l 4}(\lambda)^{-1}=-A_{r 1}(\lambda)^{-1} A_{r 2}(\lambda), \\
& \tilde{R}(\lambda)=A_{r 3}(\lambda) A_{r 1}(\lambda)^{-1}=-A_{l 4}(\lambda)^{-1} A_{l 3}(\lambda),
\end{aligned}
$$

are called reflection coefficient from the right and reflection coefficient from the left, respectively. Moreover, we have the Riemann-Hilbert problem

$$
F_{+}(\lambda, x)=F_{-}(\lambda, x) J \tilde{S}(\lambda) J .
$$

In the same way we derive the Riemann-Hilbert problems

$$
\begin{aligned}
& \check{F}_{-}(\lambda, x)=S(\lambda) \check{F}_{+}(\lambda, x), \\
& \check{F}_{+}(\lambda, x)=\tilde{S}(\lambda) \check{F}_{-}(\lambda, x) .
\end{aligned}
$$

Obviously, $S(\lambda)$ and $\tilde{S}(\lambda)$ are each others inverses. In the focusing case the scattering matrices are $J$-unitary:

$$
\tilde{S}(\lambda)=S(\lambda)^{-1}=J S(\lambda)^{\dagger} J, \quad \lambda \in \mathbb{R} .
$$

In the defocusing case, where the technical hypothesis is always satisfied $[6,11]$, the scattering matrices are unitary for $\lambda \in \mathbb{R}$.

c. Fourier integral representations of reflection coefficients. Under the technical hypothesis it can easily be shown ([11]; also $[6,17]$ in the defocusing and focusing cases) that there exist matrix functions $\hat{L}(\alpha), \hat{\tilde{L}}(\alpha), \hat{R}(\alpha)$, and $\hat{\tilde{R}}(\alpha)$ having their entries in $L^{1}(\mathbb{R})$ such that

$$
\begin{array}{ll}
R(\lambda)=\int_{-\infty}^{\infty} d \alpha e^{-i \lambda \alpha} \hat{R}(\alpha), & \tilde{R}(\lambda)=\int_{-\infty}^{\infty} d \alpha e^{i \lambda \alpha} \hat{\tilde{R}}(\alpha), \\
L(\lambda)=\int_{-\infty}^{\infty} d \alpha e^{i \lambda \alpha} \hat{L}(\alpha), & \tilde{L}(\lambda)=\int_{-\infty}^{\infty} d \alpha e^{-i \lambda \alpha} \hat{\tilde{L}}(\alpha) .
\end{array}
$$

With respect to $[6,11,17]$ we have replaced $e^{ \pm i \lambda \alpha}$ by $e^{\mp i \lambda \alpha}$ in the definitions of $\hat{L}(\alpha)$ and $\hat{\tilde{L}}(\alpha)$. 


\section{Derivation of Marchenko Integral Equations}

Before deriving the Marchenko integral equations we state the following refinement of Proposition 2.2. Although we have catered to the notations of [3], its proof can be given as in [11] (see [6] in the defocusing case and [17] in the focusing case).

PROPOSITION 5.1. Suppose the entries of $q(x)$ and $r(x)$ belong to $L^{1}(\mathbb{R})$. Then there exist matrix functions $\bar{K}(x, y), \bar{G}(x, y), G(x, y)$, and $K(x, y)$ such that

$$
\begin{aligned}
& \bar{\psi}(\lambda, x)=e^{i \lambda x}\left[\begin{array}{c}
I_{n} \\
0_{m \times n}
\end{array}\right]+\int_{x}^{\infty} d y \bar{K}(x, y) e^{i \lambda y}, \\
& \bar{\phi}(\lambda, x)=e^{-i \lambda x}\left[\begin{array}{c}
0_{n \times m} \\
I_{m}
\end{array}\right]+\int_{-\infty}^{x} d y \bar{G}(x, y) e^{-i \lambda y}, \\
& \phi(\lambda, x)=e^{i \lambda x}\left[\begin{array}{c}
I_{n} \\
0_{m \times n}
\end{array}\right]+\int_{-\infty}^{x} d y G(x, y) e^{i \lambda y}, \\
& \psi(\lambda, x)=e^{-i \lambda x}\left[\begin{array}{c}
0_{n \times m} \\
I_{m}
\end{array}\right]+\int_{x}^{\infty} d y K(x, y) e^{-i \lambda y} .
\end{aligned}
$$

Moreover, for each $x \in \mathbb{R}$ the entries of $K(x, y)$ and $\bar{K}(x, y)$ belong to $L^{1}(x,+\infty)$ and those of $G(x, y)$ and $\bar{G}(x, y)$ belong to $L^{1}(-\infty, x)$. Analogously, there exist matrix functions $\bar{G}(y, x), \breve{K}(y, x), \check{K}(y, x)$, and $\check{G}(y, x)$ such that

$$
\begin{aligned}
& \bar{\phi}(\lambda, x)=e^{-i \lambda x}\left[\begin{array}{ll}
I_{n} & 0_{n \times m}
\end{array}\right]+\int_{-\infty}^{x} d y \check{\check{G}}(y, x) e^{-i \lambda y}, \\
& \check{\psi}(\lambda, x)=e^{i \lambda x}\left[\begin{array}{ll}
0_{m \times n} & I_{m}
\end{array}\right]+\int_{x}^{\infty} d y \check{\check{K}}(y, x) e^{i \lambda y}, \\
& \check{\psi}(\lambda, x)=e^{-i \lambda x}\left[\begin{array}{ll}
I_{n} & 0_{n \times m}
\end{array}\right]+\int_{x}^{\infty} d y \check{K}(y, x) e^{-i \lambda y}, \\
& \check{\phi}(\lambda, x)=e^{i \lambda x}\left[\begin{array}{ll}
0_{m \times n} & I_{m}
\end{array}\right]+\int_{-\infty}^{x} d y \check{G}(y, x) e^{i \lambda y} .
\end{aligned}
$$

In addition, for each $x \in \mathbb{R}$ the entries of $\check{K}(y, x)$ and $\breve{K}(y, x)$ belong to $L^{1}(x,+\infty)$ and those of $\breve{G}(y, x)$ and $\bar{G}(y, x)$ belong to $L^{1}(-\infty, x)$.

We now derive the Marchenko integral equations from the Riemann-Hilbert problems (4.8), (4.10), (4.11), and (4.12), two equations for each Riemann-Hilbert problem in the absence of symmetries. We first deal with the elementary situation in which the transmission coefficients $T_{l}(\lambda)$ and $T_{r}(\lambda)$ are analytic in $\lambda \in \mathbb{C}^{+}$and the transmission coefficients $\tilde{T}_{l}(\lambda)$ and $\tilde{T}_{r}(\lambda)$ are analytic in $\lambda \in \mathbb{C}^{-}$. Next we consider the case where the poles of the four transmission coefficients all have order one and the technical hypothesis is satisfied. Finally, we treat the most general case under the technical hypothesis. Contrary to [2,3], we then assume arbitrary Jordan structure.

a. Marchenko equations if there are no bound states. We now derive the Marchenko integral equation in the absence of bound states. 
THEOREM 5.2. Under the technical hypothesis, suppose the entries of $q(x)$ and $r(x)$ belong to $L^{1}(\mathbb{R})$ and the Hamiltonian does not have any nonreal eigenvalues. Then the following Marchenko integral equations are true:

$$
\begin{array}{r}
\bar{K}(x, y)+\left[\begin{array}{c}
0_{n \times m} \\
I_{m}
\end{array}\right] \hat{\tilde{R}}(x+y)+\int_{x}^{\infty} d z K(x, z) \hat{\tilde{R}}(z+y)=0, \\
\bar{G}(x, y)+\left[\begin{array}{c}
I_{n} \\
0_{m \times n}
\end{array}\right] \hat{\tilde{L}}(x+y)+\int_{-\infty}^{x} d z G(x, z) \hat{\tilde{L}}(z+y)=0, \\
G(x, y)+\left[\begin{array}{c}
0_{n \times m} \\
I_{m}
\end{array}\right] \hat{L}(x+y)+\int_{-\infty}^{x} d z \bar{G}(x, z) \hat{L}(z+y)=0, \\
K(x, y)+\left[\begin{array}{c}
I_{n} \\
0_{m \times n}
\end{array}\right] \hat{R}(x+y)+\int_{x}^{\infty} d z \bar{K}(x, z) \hat{R}(z+y)=0, \\
\bar{G}(y, x)-\hat{\tilde{L}}(y+x)\left[\begin{array}{ll}
0_{m \times n} & I_{m}
\end{array}\right]-\int_{-\infty}^{x} d z \hat{\tilde{L}}(y+z) \check{G}(z, x)=0, \\
\bar{K}(y, x)-\hat{\tilde{R}}(y+x)\left[\begin{array}{ll}
I_{n} & 0_{n \times m}
\end{array}\right]-\int_{x}^{\infty} d z \hat{\tilde{R}}(y+z) \check{K}(z, x)=0, \\
\check{K}(y, x)-\hat{R}(y+x)\left[\begin{array}{ll}
0_{m \times n} & I_{m}
\end{array}\right]-\int_{x}^{\infty} d z \hat{R}(y+z) \bar{K}(z, x)=0, \\
\check{G}(y, x)-\hat{L}(y+x)\left[\begin{array}{ll}
I_{n} & 0_{n \times m}
\end{array}\right]-\int_{-\infty}^{x} d z \hat{L}(y+z) \bar{G}(z, x)=0 .
\end{array}
$$

Proof. Let us sketch the proof of (5.2a). The left $n$ columns of (4.10) can be written as

$$
e^{-i \lambda x} \bar{\psi}(\lambda, x)-e^{-i \lambda x} \phi(\lambda, x) \tilde{T}_{l}(\lambda)+e^{i \lambda x} \psi(\lambda, x) e^{-2 i \lambda x} \tilde{R}(\lambda)=0,
$$

where the first term is continuous in $\overline{\mathbb{C}^{+}}$and analytic in $\mathbb{C}^{+}$, the second is continuous in $\overline{\mathbb{C}^{-}}$and analytic in $\mathbb{C}^{-}$(because $\tilde{T}_{l}(\lambda)$ is assumed to have no poles in $\mathbb{C}^{-}$), and the third is continuous in $\lambda \in \mathbb{R}$ and vanishes as $\lambda \rightarrow \pm \infty$. Similarly we have the Riemann-Hilbert problems

$$
\begin{array}{r}
e^{i \lambda x} \bar{\phi}(\lambda, x)-e^{i \lambda x} \psi(\lambda, x) \tilde{T}_{r}(\lambda)+e^{-i \lambda x} \phi(\lambda, x) e^{2 i \lambda x} \tilde{L}(\lambda)=0, \\
e^{-i \lambda x} \phi(\lambda, x)-e^{-i \lambda x} \bar{\psi}(\lambda, x) T_{l}(\lambda)+e^{i \lambda x} \bar{\phi}(\lambda, x) e^{-2 i \lambda x} L(\lambda)=0, \\
e^{i \lambda x} \psi(\lambda, x)-e^{i \lambda x} \bar{\phi}(\lambda, x) T_{r}(\lambda)+e^{-i \lambda x} \bar{\psi}(\lambda, x) e^{2 i \lambda x} R(\lambda)=0, \\
e^{i \lambda x} \bar{\phi}(\lambda, x)-\tilde{T}_{l}(\lambda) e^{i \lambda x} \check{\psi}(\lambda, x)-e^{2 i \lambda x} \tilde{L}(\lambda) e^{-i \lambda x} \check{\phi}(\lambda, x)=0,
\end{array}
$$




$$
\begin{aligned}
& e^{-i \lambda x} \bar{\psi}(\lambda, x)-\tilde{T}_{r}(\lambda) e^{-i \lambda x} \check{\phi}(\lambda, x)-e^{-2 i \lambda x} \tilde{R}(\lambda) e^{i \lambda x} \check{\psi}(\lambda, x)=0, \\
& e^{i \lambda x} \check{\psi}(\lambda, x)-T_{l}(\lambda) e^{i \lambda x \bar{\phi}}(\lambda, x)-e^{2 i \lambda x} R(\lambda) e^{-i \lambda x} \overline{\check{\psi}}(\lambda, x)=0, \\
& e^{-i \lambda x} \check{\phi}(\lambda, x)-T_{r}(\lambda) e^{-i \lambda x} \overline{\check{\psi}}(\lambda, x)-e^{-2 i \lambda x} L(\lambda) e^{i \lambda x} \bar{\varnothing}(\lambda, x)=0,
\end{aligned}
$$

where we have used the last $m$ columns of (4.10), the first $n$ and last $m$ columns of (4.8), the first $n$ and the last $m$ rows of (4.12), and the first $n$ and the last $m$ rows of (4.11), respectively. By inverting the Fourier transforms in (5.1) we now obtain the respective identities

$$
\begin{aligned}
& \bar{K}(x, y)=\frac{1}{2 \pi} \int_{-\infty}^{\infty} d \lambda\left\{e^{-i \lambda x} \bar{\psi}(\lambda, x)-\left[\begin{array}{c}
I_{n} \\
0_{m \times n}
\end{array}\right]\right\} e^{i \lambda(x-y)} . \\
& \bar{G}(x, y)=\frac{1}{2 \pi} \int_{-\infty}^{\infty} d \lambda\left\{e^{i \lambda x} \bar{\phi}(\lambda, x)-\left[\begin{array}{c}
0_{n \times m} \\
I_{m}
\end{array}\right]\right\} e^{i \lambda(y-x)} . \\
& G(x, y)=\frac{1}{2 \pi} \int_{-\infty}^{\infty} d \lambda\left\{e^{-i \lambda x} \phi(\lambda, x)-\left[\begin{array}{c}
I_{n} \\
0_{m \times n}
\end{array}\right]\right\} e^{i \lambda(x-y)} . \\
& K(x, y)=\frac{1}{2 \pi} \int_{-\infty}^{\infty} d \lambda\left\{e^{i \lambda x} \psi(\lambda, x)-\left[\begin{array}{c}
0_{n \times m} \\
I_{m}
\end{array}\right]\right\} e^{i \lambda(y-x)} .
\end{aligned}
$$

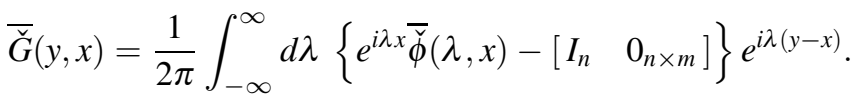

$$
\begin{aligned}
& \overline{\check{K}}(y, x)=\frac{1}{2 \pi} \int_{-\infty}^{\infty} d \lambda\left\{e^{-i \lambda x} \overline{\check{\psi}}(\lambda, x)-\left[\begin{array}{ll}
0_{m \times n} & I_{m}
\end{array}\right]\right\} e^{i \lambda(x-y)} \\
& \check{K}(y, x)=\frac{1}{2 \pi} \int_{-\infty}^{\infty} d \lambda\left\{e^{i \lambda x} \check{\psi}(\lambda, x)-\left[\begin{array}{ll}
I_{n} & 0_{n \times m}
\end{array}\right]\right\} e^{i \lambda(y-x)} . \\
& \check{G}(y, x)=\frac{1}{2 \pi} \int_{-\infty}^{\infty} d \lambda\left\{e^{-i \lambda x} \check{\phi}(\lambda, x)-\left[\begin{array}{ll}
0_{m \times n} & I_{m}
\end{array}\right]\right\} e^{i \lambda(x-y)} .
\end{aligned}
$$

Applying the Fourier transformation in $(5.4 \mathrm{a})$ we obtain for the third term in $(5.3 \mathrm{a})$

$$
\begin{aligned}
\frac{1}{2 \pi} \int_{-\infty}^{\infty} d \lambda e^{i \lambda(x-y)} \int_{x}^{\infty} d z K(x, z) e^{i \lambda(x-z)} \int_{-\infty}^{\infty} d \alpha \hat{R}(\alpha) e^{i \lambda(\alpha-2 x)} \\
=\int_{x}^{\infty} d z K(x, z) \int_{-\infty}^{\infty} d \alpha \hat{R}(\alpha) \frac{1}{2 \pi} \int_{-\infty}^{\infty} d \lambda e^{i \lambda(\alpha-y-z)} \\
=\int_{x}^{\infty} d z K(x, z) \int_{-\infty}^{\infty} d \alpha \hat{R}(\alpha) \delta(\alpha-y-z)=\int_{x}^{\infty} d z K(x, z) \hat{R}(z+y)
\end{aligned}
$$

which implies (5.2a). Equations (5.2b)-(5.2h) are proved likewise.

b. Marchenko equations when the geometric and algebraic multiplicities of the eigenvalues coincide. We first describe the eigenspaces of the Hamiltonian $H$ in terms of the residues of the transmission coefficients when their algebraic and 
geometric multiplicity coincide (i.e., if the transmission coefficients have only simple poles: $\left.Q_{j}=1\right)$. We then go on to derive the eight Marchenko integral equations.

LEMMA 5.3. If $\lambda_{j} \in \mathbb{C}^{+}$and $Q_{j}=1$, then

$$
\operatorname{Ker} A_{l 1}\left(\lambda_{j}\right)=\left\{\tau_{l j 0} \xi: \xi \in \mathbb{C}^{n}\right\}, \quad \operatorname{Ker} A_{r 4}\left(\lambda_{j}\right)=\left\{\tau_{r j 0} \zeta: \zeta \in \mathbb{C}^{m}\right\} .
$$

Analogously, if $\tilde{\lambda}_{j} \in \mathbb{C}^{-}$and $\tilde{Q}_{j}=1$, then

$\operatorname{Ker} A_{r 1}\left(\tilde{\lambda}_{j}\right)=\left\{\tilde{\tau}_{r j 0} \xi: \xi \in \mathbb{C}^{n}\right\}, \quad \operatorname{Ker} A_{l 4}\left(\tilde{\lambda}_{j}\right)=\left\{\tilde{\tau}_{l j 0} \zeta: \zeta \in \mathbb{C}^{m}\right\}$.

Proof. Let $A_{l 1}\left(\lambda_{j}\right) \varepsilon_{j}=0$ for some $0 \neq \varepsilon_{j} \in \mathbb{C}^{n}$. Then

$$
A_{l 1}(\lambda) \varepsilon_{j}=\left(\lambda-\lambda_{j}\right) f(\lambda)
$$

for some vector function $f(\lambda)$ that is analytic in a neighborhood of $\lambda$. Using (4.1) to write $\varepsilon_{j}=\left(\lambda-\lambda_{j}\right) T_{l}(\lambda) f(\lambda)$ and taking the limit as $\lambda \rightarrow \lambda_{j}$, we obtain from (4.3) that $\varepsilon_{j}=i \tau_{l j 0} f\left(\lambda_{j}\right)$, which proves that $\varepsilon_{j}$ belongs to the range of $\tau_{l j 0}$. Conversely, if $\varepsilon=\tau_{l j 0} \xi$ for some $\xi \in \mathbb{C}^{n}$, then for some matrix function $T_{l j}(\lambda)$ analytic in a neighborhood of $\lambda_{j}$ and vanishing at $\lambda_{j}$ we have

$$
A_{l 1}(\lambda) \varepsilon_{j}=-i A_{l 1}(\lambda)\left[\left(\lambda-\lambda_{j}\right) T_{l}(\lambda) \xi-T_{l j}(\lambda) \xi\right]=-i\left(\lambda-\lambda_{j}\right) \xi+i T_{l j}(\lambda) \xi \rightarrow 0_{n \times 1}
$$

as $\lambda \rightarrow \lambda_{j}$, so that $A_{l 1}\left(\lambda_{j}\right) \varepsilon_{j}=0_{n \times 1}$. Consequently, we obtain the first of (5.5). The second of (5.5) and both of (5.6) are proved in the same way.

Recall the two representations of the eigenfunctions and dual eigenfunctions for eigenvalues $\lambda_{j} \in \mathbb{C}^{+}$and $\tilde{\lambda}_{j} \in \mathbb{C}^{-}$[cf. (3.3)-(3.4), (3.9)-(3.10), (3.11)-(3.12), and (3.13)-(3.14)]. We then introduce the norming constant matrices $\Gamma_{l j 0}, \Gamma_{r j 0}, \tilde{\Gamma}_{l j 0}, \tilde{\Gamma}_{r j 0}$, $\check{\Gamma}_{l j 0}, \check{\Gamma}_{r j 0}, \widetilde{\check{\Gamma}}_{l j 0}$, and $\tilde{\check{\Gamma}}_{r j 0}$ as follows:

$$
\begin{aligned}
& \bar{\psi}\left(\lambda_{j}, x\right) \tau_{l j 0}=\bar{\phi}\left(\lambda_{j}, x\right) \Gamma_{r j 0}, \quad \bar{\phi}\left(\lambda_{j}, x\right) \tau_{r j 0}=\bar{\psi}\left(\lambda_{j}, x\right) \Gamma_{l j 0}, \\
& \phi\left(\tilde{\lambda}_{j}, x\right) \tilde{\tau}_{l j 0}=\psi\left(\tilde{\lambda}_{j}, x\right) \tilde{\Gamma}_{l j 0}, \quad \psi\left(\tilde{\lambda}_{j}, x\right) \tilde{\tau}_{r j 0}=\phi\left(\tilde{\lambda}_{j}, x\right) \tilde{\Gamma}_{r j 0}, \\
& \tau_{l j 0} \bar{\phi}\left(\lambda_{j}, x\right)=\check{\Gamma}_{l j 0} \bar{\psi}\left(\lambda_{j}, x\right), \quad \tau_{r j 0} \bar{\psi}\left(\lambda_{j}, x\right)=\check{\Gamma}_{r j 0} \bar{\phi}\left(\lambda_{j}, x\right), \\
& \tilde{\tau}_{l j 0} \check{\psi}\left(\tilde{\lambda}_{j}, x\right)=\tilde{\Gamma}_{r j 0} \check{\phi}\left(\tilde{\lambda}_{j}, x\right), \quad \tilde{\tau}_{r j 0} \check{\phi}\left(\tilde{\lambda}_{j}, x\right)=\check{\Gamma}_{l j 0} \check{\psi}\left(\tilde{\lambda}_{j}, x\right),
\end{aligned}
$$

where the ranges of $\Gamma_{l j 0}, \Gamma_{r j 0}, \tilde{\Gamma}_{l j 0}$, and $\tilde{\Gamma}_{r j 0}$ coincide with those of $\tau_{l j 0}, \tau_{r j 0}, \tilde{\tau}_{r j 0}$, and $\tilde{\tau}_{l j 0}$, respectively, and the kernels of $\check{\Gamma}_{l j 0}, \check{\Gamma}_{r j 0}, \tilde{\Gamma}_{l j 0}$, and $\tilde{\Gamma}_{r j 0}$ with those of $\check{\tau}_{l j 0}$, $\check{\tau}_{r j 0}, \tilde{\widetilde{\tau}}_{r j 0}$, and $\tilde{\widetilde{\tau}}_{l j 0}$, respectively.

By the above constructions (5.7) we avoid introducing dependency constant matrices which relate vectors as $\varepsilon$ and $\eta$ in (3.1). In the matrix Zakharov-Shabat case, such relations are partially defined matrices which then have to be extended to full matrices by defining them as zero on the orthogonal complements of their original domains. This has in fact been accomplished in [11]. By defining norming constant matrices directly we avoid the major hassle of having to introduce matrices that do not even appear in the Marchenko integral kernels.

Let us now proceed to deriving the Marchenko integral equations. 
THEOREM 5.4. Under the technical hypothesis, suppose the entries of $q(x)$ and $r(x)$ belong to $L^{1}(\mathbb{R})$ and the resolvent of the Hamiltonian only has simple poles. Then the following Marchenko integral equations are true:

$$
\begin{array}{r}
\bar{K}(x, y)+\left[\begin{array}{c}
0_{n \times m} \\
I_{m}
\end{array}\right] \tilde{\Omega}_{l}(x+y)+\int_{x}^{\infty} d z K(x, z) \tilde{\Omega}_{l}(z+y)=0, \\
\bar{G}(x, y)+\left[\begin{array}{c}
I_{n} \\
0_{m \times n}
\end{array}\right] \tilde{\Omega}_{r}(x+y)+\int_{-\infty}^{x} d z G(x, z) \tilde{\Omega}_{r}(z+y)=0, \\
G(x, y)+\left[\begin{array}{c}
0_{n \times m} \\
I_{m}
\end{array}\right] \Omega_{r}(x+y)+\int_{-\infty}^{x} d z \bar{G}(x, z) \Omega_{r}(z+y)=0, \\
K(x, y)+\left[\begin{array}{c}
I_{n} \\
0_{m \times n}
\end{array}\right] \Omega_{l}(x+y)+\int_{x}^{\infty} d z \bar{K}(x, z) \Omega_{l}(z+y)=0, \\
\bar{G}(y, x)-\check{\Omega}_{r}(y+x)\left[\begin{array}{ll}
0_{m \times n} & I_{m}
\end{array}\right]-\int_{-\infty}^{x} d z \check{\Omega}_{r}(y+z) \check{G}(z, x)=0, \\
\bar{K}(y, x)-\check{\Omega}_{l}(y+x)\left[\begin{array}{ll}
I_{n} & 0_{n \times m}
\end{array}\right]-\int_{x}^{\infty} d z \check{\Omega}_{l}(y+z) \check{K}(z, x)=0, \\
\check{K}(y, x)-\check{\Omega}_{l}(y+x)\left[\begin{array}{ll}
0_{m \times n} & I_{m}
\end{array}\right]-\int_{x}^{\infty} d z \check{\Omega}_{l}(y+z) \bar{K}(z, x)=0, \\
\check{G}(y, x)-\check{\Omega}_{r}(y+x)\left[\begin{array}{ll}
I_{n} & 0_{n \times m}
\end{array}\right]-\int_{-\infty}^{x} d z \check{\Omega}_{r}(y+z) \check{G}(z, x)=0,
\end{array}
$$

where

$$
\begin{array}{ll}
\tilde{\Omega}_{l}(w)=\hat{\tilde{R}}(w)+\sum_{j} e^{-i \tilde{\lambda}_{j} w} \tilde{\Gamma}_{l j 0}, & \tilde{\Omega}_{r}(w)=\hat{\tilde{L}}(w)+\sum_{j} e^{i \tilde{\lambda}_{j} w} \tilde{\Gamma}_{r j 0}, \\
\Omega_{r}(w)=\hat{L}(w)+\sum_{j} e^{-i \lambda_{j} w} \Gamma_{r j 0}, & \Omega_{l}(w)=\hat{R}(w)+\sum_{j} e^{i \lambda_{j} w} \Gamma_{l j 0}, \\
\check{\Omega}_{r}(w)=\hat{\tilde{L}}(w)+\sum_{j} e^{i \tilde{\lambda}_{j} w} \tilde{\Gamma}_{r j 0}, & \check{\Omega}_{l}(w)=\hat{\tilde{R}}(w)+\sum_{j} e^{-i \tilde{\lambda}_{j} w} \tilde{\Gamma}_{l j 0}, \\
\check{\Omega}_{l}(w)=\hat{R}(w)+\sum_{j} e^{i \lambda_{j} w \check{\Gamma}_{l j 0},} & \check{\Omega}_{r}(w)=\hat{L}(w)+\sum_{j} e^{-i \lambda_{j} w} \check{\Gamma}_{r j 0} .
\end{array}
$$

Proof. To prove (5.8a), we apply the Fourier transform in (5.4a) to the second term in (5.3a), use the first of (5.7b), employ (5.1d) for $\lambda=\tilde{\lambda}_{j}$, and get

$$
\begin{gathered}
-\frac{1}{2 \pi} \int_{-\infty}^{\infty} d \lambda e^{-i \lambda y} \phi(\lambda, x) \frac{-i \tilde{\tau}_{l j 0}}{\lambda-\tilde{\lambda}_{j}}=-e^{-i \tilde{\lambda}_{j y} y} \phi\left(\tilde{\lambda}_{j}, x\right) \tilde{\tau}_{l j 0}=-e^{-i \tilde{\lambda}_{j y} y} \psi\left(\tilde{\lambda}_{j}, x\right) \tilde{\Gamma}_{l j 0} \\
=-e^{-i \tilde{\lambda}_{j}(x+y)}\left[\begin{array}{c}
0_{n \times m} \\
I_{m}
\end{array}\right] \tilde{\Gamma}_{l j 0}-\int_{x}^{\infty} d z e^{-i \tilde{\lambda}_{j}(z+y)} K(x, z) \tilde{\Gamma}_{l j 0},
\end{gathered}
$$


which implies (5.8a). Similarly, applying the Fourier transform in (5.4b) to the second term in $(5.3 \mathrm{~b})$, using the second of $(5.7 \mathrm{~b})$, and $(5.1 \mathrm{~b})$, we get

$$
\begin{gathered}
-\frac{1}{2 \pi} \int_{-\infty}^{\infty} d \lambda e^{i \lambda y} \psi(\lambda, x) \frac{-i \tilde{\tau}_{r j 0}}{\lambda-\tilde{\lambda}_{j}}=-e^{i \tilde{\lambda}_{j y} y} \psi\left(\tilde{\lambda}_{j}, x\right) \tilde{\tau}_{r j 0}=-e^{i \tilde{\lambda}_{j y} y} \phi\left(\tilde{\lambda}_{j}, x\right) \tilde{\Gamma}_{r j 0} \\
=-e^{i \tilde{\lambda}_{j}(x+y)}\left[\begin{array}{c}
I_{n} \\
0_{m \times n}
\end{array}\right] \tilde{\Gamma}_{r j 0}-\int_{-\infty}^{x} d z e^{i \tilde{\lambda}_{j}(z+y)} G(x, z) \tilde{\Gamma}_{r j 0},
\end{gathered}
$$

which implies (5.8b). To prove (5.8c), we apply the inverse Fourier transform in $(5.4 \mathrm{c})$ to the second term in (5.3c), use the first of (5.7a), substitute (5.1b) for $\lambda=\lambda_{j}$, and get

$$
\begin{gathered}
-\frac{1}{2 \pi} \int_{-\infty}^{\infty} d \lambda e^{-i \lambda y} \bar{\psi}(\lambda, x) \frac{i \tau_{l j 0}}{\lambda-\lambda_{j}}=e^{-i \lambda_{j} y} \bar{\psi}\left(\lambda_{j}, x\right) \tau_{l j 0}=e^{-i \lambda_{j} y} \bar{\phi}\left(\lambda_{j}, x\right) \Gamma_{r j 0} \\
=e^{-i \lambda_{j}(x+y)}\left[\begin{array}{c}
0_{n \times m} \\
I_{m}
\end{array}\right] \Gamma_{r j 0}+\int_{-\infty}^{x} d z \bar{G}(x, z) e^{-i \lambda_{j}(z+y)} \Gamma_{r j 0},
\end{gathered}
$$

which implies (5.8c). Applying the inverse Fourier transform in (5.4d) to the second term in (5.3d), use the second of (5.7a), substitute (5.1a) for $\lambda=\lambda_{j}$, we get

$$
\begin{gathered}
-\frac{1}{2 \pi} \int_{-\infty}^{\infty} d \lambda e^{i \lambda y} \bar{\phi}(\lambda, x) \frac{i \tau_{r j 0}}{\lambda-\lambda_{j}}=e^{i \lambda_{j y} y} \bar{\phi}\left(\lambda_{j}, x\right) \tau_{r j 0}=e^{i \lambda_{j} y} \bar{\psi}\left(\lambda_{j}, x\right) \Gamma_{l j 0} \\
=e^{i \lambda_{j}(x+y)}\left[\begin{array}{c}
I_{n} \\
0_{m \times n}
\end{array}\right] \Gamma_{l j 0}+\int_{x}^{\infty} d z \bar{K}(x, z) e^{i \lambda_{j}(z+y)} \Gamma_{l j 0},
\end{gathered}
$$

which implies (5.8d). Applying the inverse Fourier transform in $(5.4 \mathrm{e})$ to the second term in $(5.3 \mathrm{e})$, use the first of $(5.7 \mathrm{~d})$, substitute $(5.1 \mathrm{~h})$ for $\lambda=\tilde{\lambda}_{j}$, we get

$$
\begin{gathered}
-\frac{1}{2 \pi} \int_{-\infty}^{\infty} d \lambda e^{i \lambda y} \frac{-i \tilde{\tau}_{l j 0}}{\lambda-\tilde{\lambda}_{j}} \check{\psi}(\lambda, x)=-e^{i \tilde{\lambda}_{j y} y} \tilde{\tau}_{l j 0} \check{\psi}\left(\tilde{\lambda}_{j}, x\right)=-e^{i \tilde{\lambda}_{j} y} \tilde{\Gamma}_{r j 0} \check{\phi}_{(}\left(\tilde{\lambda}_{j}, x\right) \\
=-e^{i \tilde{\lambda}_{j}(x+y)} \tilde{\Sigma}_{r j 0}\left[\begin{array}{ll}
0_{m \times n} & I_{m}
\end{array}\right]-\int_{-\infty}^{x} d z e^{i \tilde{\lambda}_{j}(z+y)} \tilde{\Gamma}_{r j 0} \tilde{G}(z, x),
\end{gathered}
$$

which implies (5.8e). Applying the inverse Fourier transform in (5.4f) to the second term in (5.3f), use the second of $(5.7 \mathrm{~d})$, substitute $(5.1 \mathrm{~g})$ for $\lambda=\tilde{\lambda}_{j}$, we get

$$
\begin{gathered}
-\frac{1}{2 \pi} \int_{-\infty}^{\infty} d \lambda e^{-i \lambda y} \frac{-i \tilde{\tau}_{r j 0}}{\lambda-\tilde{\lambda}_{j}} \check{\phi}(\lambda, x)=-e^{-i \tilde{\lambda}_{j} y} \tilde{\tau}_{r j 0} \check{\phi}\left(\tilde{\lambda}_{j}, x\right)=-e^{-i \tilde{\lambda}_{j} y} \tilde{\check{\Gamma}}_{l j 0} \check{\psi}^{\left(\tilde{\lambda}_{j}, x\right)} \\
=-e^{-i \tilde{\lambda}_{j}(x+y)} \tilde{\Gamma}_{l j 0}\left[\begin{array}{ll}
I_{n} & 0_{n \times m}
\end{array}\right]-\int_{x}^{\infty} d z e^{-i \tilde{\lambda}_{j}(z+y)} \tilde{\Gamma}_{l j 0} \tilde{K}(z, x),
\end{gathered}
$$

which implies (5.8f). Applying the inverse Fourier transform in $(5.4 \mathrm{~g})$ to the second term in $(5.3 \mathrm{~g})$, use the first of $(5.7 \mathrm{c})$ and $(5.1 \mathrm{f})$, we get

$$
\begin{aligned}
& -\frac{1}{2 \pi} \int_{-\infty}^{\infty} d \lambda e^{i \lambda y} \frac{i \tau_{l j 0}}{\lambda-\lambda_{j}} \bar{\phi}(\lambda, x)=e^{i \lambda_{j} y} \tau_{l j 0} \check{\varnothing}\left(\lambda_{j}, x\right)=e^{i \lambda_{j} y} \check{\Gamma}_{l j 0} \bar{\psi} \check{\psi}\left(\lambda_{j}, x\right) \\
& =e^{i \lambda_{j}(x+y)} \check{\Gamma}_{l j 0}\left[\begin{array}{ll}
0_{m \times n} & I_{m}
\end{array}\right]+\int_{x}^{\infty} d z e^{i \lambda_{j}(z+y)} \check{\Gamma}_{l j 0} \check{K}(z, x),
\end{aligned}
$$


which implies $(5.8 \mathrm{~g})$. Finally, applying the inverse Fourier transform in $(5.4 \mathrm{~h})$ to the second term in $(5.3 \mathrm{~h})$, use the second of $(5.7 \mathrm{c})$ and $(5.1 \mathrm{e})$, we get

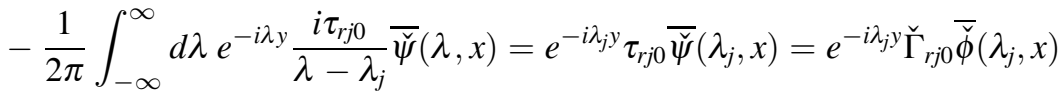

$$
\begin{aligned}
& =e^{-i \lambda_{j}(x+y)} \check{\Gamma}_{r j 0}\left[\begin{array}{ll}
I_{n} & 0_{n \times m}
\end{array}\right]+\int_{-\infty}^{x} d z e^{-i \lambda_{j}(z+y)} \check{\Gamma}_{r j 0} \check{\breve{G}}(z, x),
\end{aligned}
$$

which implies (5.8h).

c. Marchenko equations for general Jordan structure. To generalize Lemma 5.3 to the case of an eigenvalue with nontrivial Jordan structure, we convert the transmission functions to locally analytic functions as follows:

$$
\begin{aligned}
& T_{l j}^{\#}(\lambda)=\left(\lambda-\lambda_{j}\right)^{Q_{j}} T_{l}(\lambda)=\sum_{s=0}^{Q_{j}-1}\left(\lambda-\lambda_{j}\right)^{s} i^{Q_{j}-s} \tau_{l j, Q_{j}-s-1}+O\left(\left(\lambda-\lambda_{j}\right)^{Q_{j}}\right), \\
& T_{r j}^{\#}(\lambda)=\left(\lambda-\lambda_{j}\right)^{Q_{j}} T_{r}(\lambda)=\sum_{s=0}^{Q_{j}-1}\left(\lambda-\lambda_{j}\right)^{s} i^{Q_{j}-s} \tau_{r j, Q_{j}-s-1}+O\left(\left(\lambda-\lambda_{j}\right)^{Q_{j}}\right),
\end{aligned}
$$

where $Q_{j}$ is the pole order of the transmission coefficients $T_{l}(\lambda)$ and $T_{r}(\lambda)$ at the eigenvalue $\lambda_{j} \in \mathbb{C}^{+}$. Analogously,

$$
\begin{aligned}
& \tilde{T}_{l j}^{\#}(\lambda)=\left(\lambda-\tilde{\lambda}_{j}\right)^{\tilde{Q}_{j}} \tilde{T}_{l}(\lambda)=\sum_{s=0}^{\tilde{Q}_{j}-1}\left(\lambda-\tilde{\lambda}_{j}\right)^{s}(-i)^{\tilde{Q}_{j}-s} \tilde{\tau}_{l j, \tilde{Q}_{j}-s-1}+O\left(\left(\lambda-\tilde{\lambda}_{j}\right)^{\tilde{Q}_{j}}\right), \\
& \tilde{T}_{r j}^{\#}(\lambda)=\left(\lambda-\tilde{\lambda}_{j}\right)^{\tilde{Q}_{j}} \tilde{T}_{r}(\lambda)=\sum_{s=0}^{\tilde{Q}_{j}-1}\left(\lambda-\tilde{\lambda}_{j}\right)^{s}(-i)^{\tilde{Q}_{j}-s} \tilde{\tau}_{r j, \tilde{Q}_{j}-s-1}+O\left(\left(\lambda-\tilde{\lambda}_{j}\right)^{\tilde{Q}_{j}}\right),
\end{aligned}
$$

where $\tilde{Q}_{j}$ is the pole order of the transmission coefficients $\tilde{T}_{l}(\lambda)$ and $\tilde{T}_{r}(\lambda)$ at the eigenvalue $\tilde{\lambda}_{j} \in \mathbb{C}^{-}$.

THEOREM 5.5. Let $\lambda_{j}$ be an eigenvalue in $\mathbb{C}^{+}$and let $s=1, \ldots, Q_{j}$. Then the vectors $\left\{\varepsilon_{0}, \varepsilon_{1}, \ldots, \varepsilon_{s-1}\right\}$ generate a Jordan chain of $A_{l 1}(\lambda)$ at $\lambda_{j}$ if and only if

$$
\left[\begin{array}{c}
0_{n\left(Q_{j}-s\right) \times 1} \\
\varepsilon_{0} \\
\vdots \\
\varepsilon_{s-1}
\end{array}\right] \in \operatorname{Im} \Xi_{Q_{j}}\left(T_{l j}^{\#} ; \lambda_{j}\right) .
$$

Similarly, $\left\{\eta_{0}, \eta_{1} \ldots, \eta_{s-1}\right\}$ generate a Jordan chain of $A_{r 4}(\lambda)$ at $\lambda_{j}$ if and only if

$$
\left[\begin{array}{c}
0_{m\left(Q_{j}-s\right) \times 1} \\
\eta_{0} \\
\vdots \\
\eta_{s-1}
\end{array}\right] \in \operatorname{Im} \Xi_{Q_{j}}\left(T_{r j}^{\#} ; \lambda_{j}\right) .
$$


Similar results hold for the eigenvalues in $\mathbb{C}^{-}$.

Proof. Let $\left\{\varepsilon_{0}, \varepsilon_{1}, \ldots, \varepsilon_{s-1}\right\}$ generate a Jordan chain of $A_{l 1}(\lambda)$ at the eigenvalue $\lambda_{j}$. Then there exists a vector function $f(\lambda)$ analytic in a neighborhood of $\lambda_{j}$ such that for $\lambda$ near $\lambda_{j}$

$$
A_{l 1}(\lambda) \sum_{\sigma=0}^{s-1}\left(\lambda-\lambda_{j}\right)^{\sigma} \varepsilon_{\sigma}=\left(\lambda-\lambda_{j}\right)^{s} f(\lambda) .
$$

Multiplying by $T_{l j}^{\#}(\lambda)=\left(\lambda-\lambda_{j}\right)^{Q_{j}} A_{l 1}(\lambda)^{-1}$ we obtain in some neighborhood of $\lambda_{j}$

$$
\sum_{\sigma=Q_{j}-s}^{Q_{j}-1}\left(\lambda-\lambda_{j}\right)^{\sigma} \varepsilon_{\sigma+s-Q_{j}}=T_{l j}^{\#}(\lambda) f(\lambda),
$$

which implies (5.10).

Conversely, assume (5.10). Then there exist $h_{0}, h_{1}, \ldots, h_{Q_{j}-1} \in \mathbb{C}^{n \times 1}$ such that

$$
\Xi_{Q_{j}}\left(T_{l j}^{\#} ; \lambda_{j}\right)\left[\begin{array}{c}
h_{0} \\
h_{1} \\
\vdots \\
\vdots \\
h_{Q_{j}-2} \\
h_{Q_{j}-1}
\end{array}\right]=\left[\begin{array}{c}
0_{n \times 1} \\
\vdots \\
0_{n \times 1} \\
\varepsilon_{0} \\
\vdots \\
\varepsilon_{s-1}
\end{array}\right]=\left[\begin{array}{c}
0_{n\left(Q_{j}-s\right) \times 1} \\
\varepsilon_{0} \\
\vdots \\
\varepsilon_{s-1}
\end{array}\right] .
$$

Writing $f(\lambda)=\sum_{\sigma=0}^{Q_{j}-1}\left(\lambda-\lambda_{j}\right)^{\sigma} h_{\sigma}$, we get (5.13) and therefore (5.12) in a neighborhood of $\lambda_{j}$. The part of the theorem involving (5.11) is proved in the same way.

Let us introduce the norming constant matrices $\Gamma_{r j s}, \Gamma_{l j s}, \check{\Gamma}_{r j s}$, and $\check{\Gamma}_{l j s}(s=$ $\left.0,1, \ldots, Q_{j}-1\right)$ connected to each discrete eigenvalue $\lambda_{j} \in \mathbb{C}^{+}$as the coefficients of the respective matrix polynomials $\gamma_{r j}(\lambda), \gamma_{l j}(\lambda), \check{\gamma}_{r j}(\lambda)$, and $\check{\gamma}_{l j}(\lambda)$ :

$$
\begin{aligned}
\gamma_{r j}(\lambda) & =\sum_{s=0}^{Q_{j}-1}\left(\lambda-\lambda_{j}\right)^{s} \Gamma_{r j s}, & \gamma_{l j}(\lambda) & =\sum_{s=0}^{Q_{j}-1}\left(\lambda-\lambda_{j}\right)^{s} \Gamma_{l j s}, \\
\check{\gamma}_{r j}(\lambda) & =\sum_{s=0}^{Q_{j}-1}\left(\lambda-\lambda_{j}\right)^{s} \check{\Gamma}_{r j s}, & \check{\gamma}_{l j}(\lambda) & =\sum_{s=0}^{Q_{j}-1}\left(\lambda-\lambda_{j}\right)^{s} \check{\Gamma}_{l j s} .
\end{aligned}
$$

Similarly, we have the relations

$$
\begin{aligned}
\tilde{\gamma}_{r j}(\lambda)=\sum_{s=0}^{Q_{j}-1}\left(\lambda-\tilde{\lambda}_{j}\right)^{s} \tilde{\Gamma}_{r j s}, & \tilde{\gamma}_{l j}(\lambda)=\sum_{s=0}^{Q_{j}-1}\left(\lambda-\tilde{\lambda}_{j}\right)^{s} \tilde{\Gamma}_{l j s}, \\
\tilde{\tilde{\gamma}}_{r j}(\lambda)=\sum_{s=0}^{Q_{j}-1}\left(\lambda-\tilde{\lambda}_{j}\right)^{s} \tilde{\Gamma}_{r j s}, & \tilde{\tilde{\gamma}}_{l j}(\lambda)=\sum_{s=0}^{Q_{j}-1}\left(\lambda-\tilde{\lambda}_{j}\right)^{s} \tilde{\Gamma}_{l j s} .
\end{aligned}
$$


We now define the norming constants by requiring that the functions

$$
\begin{aligned}
& \bar{\psi}(\lambda, x) T_{l}(\lambda)-i \sum_{j} \bar{\phi}(\lambda, x) \frac{\gamma_{r j}(\lambda)}{\left(\lambda-\lambda_{j}\right)^{Q_{j}}}, \\
& \bar{\phi}(\lambda, x) T_{r}(\lambda)-i \sum_{j} \bar{\psi}(\lambda, x) \frac{\gamma_{l j}(\lambda)}{\left(\lambda-\lambda_{j}\right)^{Q_{j}}},
\end{aligned}
$$

are analytic in $\lambda \in \mathbb{C}^{+}$, the functions

$$
\begin{gathered}
\phi(\lambda, x) \tilde{T}_{l}(\lambda)+i \sum_{j} \psi(\lambda, x) \frac{\tilde{\gamma}_{l j}(\lambda)}{\left(\lambda-\tilde{\lambda}_{j}\right)}, \\
\psi(\lambda, x) \tilde{Q}_{r}(\lambda)+i \sum_{j} \phi(\lambda, x) \frac{\tilde{\gamma}_{r j}(\lambda)}{\left(\lambda-\tilde{\lambda}_{j}\right)},
\end{gathered}
$$

are analytic in $\lambda \in \mathbb{C}^{-}$, the functions

$$
\begin{aligned}
& T_{l}(\lambda) \bar{\phi}(\lambda, x)-i \sum_{j} \frac{\check{\gamma}_{l j}(\lambda)}{\left(\lambda-\lambda_{j}\right)^{Q_{j}}} \bar{\psi}(\lambda, x), \\
& T_{r}(\lambda) \bar{\psi}(\lambda, x)-i \sum_{j} \frac{\check{\gamma}_{r j}(\lambda)}{\left(\lambda-\lambda_{j}\right)^{Q_{j}}} \overline{\breve{\phi}}(\lambda, x) .
\end{aligned}
$$

are analytic in $\lambda \in \mathbb{C}^{+}$, and the functions

$$
\begin{aligned}
& \tilde{T}_{l}(\lambda) \check{\psi}(\lambda, x)+i \sum_{j} \frac{\tilde{\tilde{\gamma}}_{r}(\lambda)}{\left(\lambda-\tilde{\lambda}_{j}\right) \tilde{Q}_{j}} \check{\phi}(\lambda, x),
\end{aligned}
$$

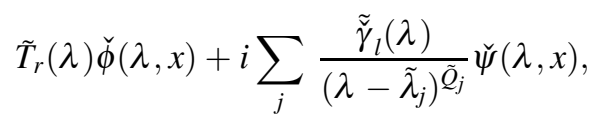

are analytic in $\lambda \in \mathbb{C}^{-}$. Equations (5.14) are a direct generalization of (5.7). Indeed, for $Q_{j}=1$ the expression (5.14a) is analytic in $\lambda \in \mathbb{C}^{+}$if and only if

$$
\lim _{\lambda \rightarrow \lambda_{j}}\left(\lambda-\lambda_{j}\right)\left(\bar{\psi}(\lambda, x) T_{l}(\lambda)-i \bar{\psi}(\lambda, x) \frac{\Gamma_{r j 0}}{\lambda-\lambda_{j}}\right)=i\left\{\bar{\psi}\left(\lambda_{j}, x\right) \tau_{l j 0}-\bar{\phi}\left(\lambda_{j}, x\right) \Gamma_{r j 0}\right\}=0
$$

which corresponds to the first of $(5.7 \mathrm{a})$. The same reasoning applies to $(5.14 \mathrm{~b})-(5.14 \mathrm{~h})$ in the case of a simple pole.

Let us multiply $(5.14 \mathrm{a})$ and $(5.14 \mathrm{f})$ by $e^{-i \lambda x}\left(\lambda-\lambda_{j}\right)^{Q_{j}},(5.14 \mathrm{c})$ and $(5.14 \mathrm{~h})$ by $e^{-i \lambda x}\left(\lambda-\lambda_{j}\right)^{\tilde{Q}_{j}},(5.14 \mathrm{~b})$ and $(5.14 \mathrm{e})$ by $e^{i \lambda x}\left(\lambda-\tilde{\lambda}_{j}\right)^{Q_{j}}$, and $(5.14 \mathrm{~d})$ and $(5.14 \mathrm{~g})$ by $e^{i \lambda x}\left(\lambda-\tilde{\lambda}_{j}\right)^{\tilde{Q}_{j}}$. Then the equations resulting from $(5.14 \mathrm{a})-(5.14 \mathrm{~b})$ and $(5.14 \mathrm{e})-(5.14 \mathrm{f})$ are $O\left(\left(\lambda-\lambda_{j}\right)^{Q_{j}}\right)$ as $\lambda \rightarrow \lambda_{j}$ and those resulting from $(5.14 \mathrm{c})-(5.14 \mathrm{~d})$ and $(5.14 \mathrm{~g})-$ $(5.14 \mathrm{~h})$ are $O\left(\left(\lambda-\tilde{\lambda}_{j}\right) \tilde{Q}_{j}\right)$ as $\lambda \rightarrow \tilde{\lambda}_{j}$. We then write the analyticity conditions in 
(5.14) in the respective forms

$$
\begin{aligned}
& \Xi_{Q_{j}}\left(e^{-i(\cdot) x} \bar{\psi}(\cdot, x) ; \lambda_{j}\right) \Xi_{Q_{j}}\left(T_{l j}^{\#} ; \lambda_{j}\right)=i \Xi_{Q_{j}}\left(e^{-2 i(\cdot) x} ; \lambda_{j}\right) \Xi_{Q_{j}}\left(e^{i(\cdot) x} \bar{\phi}(\cdot, x) ; \lambda_{j}\right) \Xi_{Q_{j}}\left(\gamma_{r j} ; \lambda_{j}\right), \\
& \Xi_{Q_{j}}\left(e^{i(\cdot) x} \bar{\phi}(\cdot, x) ; \lambda_{j}\right) \Xi_{Q_{j}}\left(T_{r j}^{\#} ; \lambda_{j}\right)=i \Xi_{Q_{j}}\left(e^{2 i(\cdot) x} ; \lambda_{j}\right) \Xi_{Q_{j}}\left(e^{-i(\cdot) x} \bar{\psi}(\cdot, x) ; \lambda_{j}\right) \Xi_{Q_{j}}\left(\gamma_{l j} ; \lambda_{j}\right) \text {, } \\
& \Xi_{\tilde{Q}_{j}}\left(e^{-i(\cdot) x} \phi(\cdot, x) ; \tilde{\lambda}_{j}\right) \Xi_{\tilde{Q}_{j}}\left(\tilde{T}_{l j}^{\#} ; \tilde{\lambda}_{j}\right)=-i \Xi_{\tilde{Q}_{j}}\left(e^{-2 i(\cdot) x} ; \tilde{\lambda}_{j}\right) \Xi_{\tilde{Q}_{j}}\left(e^{i(\cdot) x} \psi(\cdot, x) ; \tilde{\lambda}_{j}\right) \Xi_{\tilde{Q}_{j}}\left(\tilde{\gamma}_{l j} ; \tilde{\lambda}_{j}\right), \\
& \Xi_{\tilde{Q}_{j}}\left(e^{i(\cdot) x} \psi(\cdot, x) ; \tilde{\lambda}_{j}\right) \Xi_{\tilde{Q}_{j}}\left(\tilde{T}_{r j}^{\#} ; \tilde{\lambda}_{j}\right)=-i \Xi_{\tilde{Q}_{j}}\left(e^{2 i(\cdot) x} ; \tilde{\lambda}_{j}\right) \Xi_{\tilde{Q}_{j}}\left(e^{-i(\cdot) x} \phi(\cdot, x) ; \tilde{\lambda}_{j}\right) \Xi_{\tilde{Q}_{j}}\left(\tilde{\gamma}_{r j} ; \tilde{\lambda}_{j}\right) \text {, } \\
& \Xi_{Q_{j}}\left(T_{l}^{\#} ; \lambda_{j}\right) \Xi_{Q_{j}}\left(e^{i(\cdot) x} \bar{\phi}(\cdot, x) ; \lambda_{j}\right)=i \Xi_{Q_{j}}\left(\check{\gamma}_{l j} ; \lambda_{j}\right) \Xi_{Q_{j}}\left(e^{-i(\cdot) x} \overline{\check{\psi}}(\cdot, x) ; \lambda_{j}\right) \Xi_{Q_{j}}\left(e^{2 i(\cdot) x} ; \lambda_{j}\right), \\
& \Xi_{Q_{j}}\left(T_{r}^{\#} ; \lambda_{j}\right) \Xi_{Q_{j}}\left(e^{-i(\cdot) x} \overline{\breve{\psi}}(\cdot, x) ; \lambda_{j}\right)=i \Xi_{Q_{j}}\left(\check{\gamma}_{r j} ; \lambda_{j}\right) \Xi_{Q_{j}}\left(e^{i(\cdot) x} \bar{\phi}(\cdot, x) ; \lambda_{j}\right) \Xi_{Q_{j}}\left(e^{-2 i(\cdot) x} ; \lambda_{j}\right) \text {, } \\
& \Xi_{\tilde{Q}_{j}}\left(\tilde{T}_{l}^{\#} ; \tilde{\lambda}_{j}\right) \Xi_{\tilde{Q}_{j}}\left(e^{i(\cdot) x} \check{\psi}(\cdot, x) ; \tilde{\lambda}_{j}\right)=-i \Xi_{\tilde{Q}_{j}}\left(\tilde{\gamma}_{r j} ; \tilde{\lambda}_{j}\right) \Xi_{\tilde{Q}_{j}}\left(e^{-i(\cdot) x} \check{\phi}(\cdot, x) ; \tilde{\lambda}_{j}\right) \Xi_{\tilde{Q}_{j}}\left(e^{2 i(\cdot) x} ; \tilde{\lambda}_{j}\right) \text {, } \\
& \Xi_{\tilde{Q}_{j}}\left(\tilde{T}_{r}^{\#} ; \tilde{\lambda}_{j}\right) \Xi_{\tilde{Q}_{j}}\left(e^{-i(\cdot) x} \check{\phi}(\cdot, x) ; \tilde{\lambda}_{j}\right)=-i \Xi_{\tilde{Q}_{j}}\left(\tilde{\tilde{\gamma}}_{l j} ; \tilde{\lambda}_{j}\right) \Xi_{\tilde{Q}_{j}}\left(e^{i(\cdot) x} \check{\psi}(\cdot, x) ; \tilde{\lambda}_{j}\right) \Xi_{\tilde{Q}_{j}}\left(e^{-2 i(\cdot) x} ; \tilde{\lambda}_{j}\right) \text {. }
\end{aligned}
$$

Here the images of $\Xi_{Q_{j}}\left(\gamma_{r j} ; \lambda_{j}\right), \Xi_{Q_{j}}\left(\gamma_{l j} ; \lambda_{j}\right), \Xi_{Q_{j}}\left(\tilde{\gamma}_{l j} ; \tilde{\lambda}_{j}\right)$, and $\Xi_{Q_{j}}\left(\tilde{\gamma}_{r j} ; \tilde{\lambda}_{j}\right)$ coincide with those of $\Xi_{Q_{j}}\left(T_{r j}^{\#} ; \lambda_{j}\right), \Xi_{Q_{j}}\left(T_{l j}^{\#} ; \lambda_{j}\right), \Xi_{\tilde{Q}_{j}}\left(\tilde{T}_{r j}^{\#} ; \tilde{\lambda}_{j}\right)$, and $\Xi_{\tilde{Q}_{j}}\left(\tilde{T}_{l j}^{\#} ; \tilde{\lambda}_{j}\right)$, respectively. By the same token, the kernels of $\Xi_{Q_{j}}\left(\check{\gamma}_{l j} ; \lambda_{j}\right), \Xi_{Q_{j}}\left(\check{\gamma}_{r j} ; \lambda_{j}\right), \Xi_{\tilde{Q}_{j}}\left(\tilde{\tilde{\gamma}}_{r j} ; \tilde{\lambda}_{j}\right)$, and $\Xi_{\tilde{Q}_{j}}\left(\tilde{\tilde{\gamma}}_{l j} ; \tilde{\lambda}_{j}\right)$ coincide with those of $\Xi_{Q_{j}}\left(T_{r}^{\#} ; \lambda_{j}\right), \Xi_{Q_{j}}\left(T_{l}^{\#} ; \lambda_{j}\right), \Xi_{\tilde{Q}_{j}}\left(\tilde{T}_{r}^{\#} ; \tilde{\lambda}_{j}\right)$, and $\Xi_{\tilde{Q}_{j}}\left(\tilde{T}_{l}^{\#} ; \tilde{\lambda}_{j}\right)$, respectively.

We now derive the Marchenko integral equations in the most general case.

THEOREM 5.6. Under the technical hypothesis, suppose the entries of $q(x)$ and $r(x)$ belong to $L^{1}(\mathbb{R})$. Then the Marchenko integral equations $(5.8 a)-(5.8 h)$ are true, where

$$
\begin{aligned}
& \tilde{\Omega}_{l}(w)=\hat{\tilde{R}}(w)+\sum_{j} \sum_{s=0}^{\tilde{Q}_{j}-1} \frac{w^{s}}{s !} e^{-i \tilde{\lambda}_{j} w} \tilde{\Gamma}_{l j, \tilde{Q}_{j}-s-1}, \\
& \tilde{\Omega}_{r}(w)=\hat{\tilde{L}}(w)+\sum_{j} \sum_{s=0}^{\tilde{Q}_{j}-1} \frac{w^{s}}{s !} e^{i \tilde{j}_{j} w} \tilde{\Gamma}_{r j, \tilde{Q}_{j}-s-1}, \\
& \Omega_{r}(w)=\hat{L}(w)+\sum_{j} \sum_{s=0}^{Q_{j}-1} e^{-i \lambda_{j} w} \frac{w^{s}}{s !} \Gamma_{r j, Q_{j}-s-1}, \\
& \Omega_{l}(w)=\hat{R}(w)+\sum_{j} \sum_{s=0}^{Q_{j}-1} e^{i \lambda_{j} w} \frac{w^{s}}{s !} \Gamma_{l j, Q_{j}-s-1}, \\
& \tilde{\Omega}_{r}(w)=\hat{\tilde{L}}(w)+\sum_{j} \sum_{s=0}^{\tilde{Q}_{j}-1} \frac{w^{s}}{s !} e^{i \tilde{\lambda}_{j} w} \tilde{\Gamma}_{r j, \tilde{Q}_{j}-s-1},
\end{aligned}
$$




$$
\begin{aligned}
& \check{\Omega}_{l}(w)=\hat{\tilde{R}}(w)+\sum_{j} \sum_{s=0}^{\tilde{Q}_{j}-1} \frac{w^{s}}{s !} e^{-i \tilde{\lambda}_{j} w} \tilde{\Gamma}_{l j, \tilde{Q}_{j}-s-1}, \\
& \check{\Omega}_{l}(w)=\hat{R}(w)+\sum_{j} \sum_{s=0}^{Q_{j}-1} \frac{w^{s}}{s !} e^{i \lambda_{j} w} \check{\Gamma}_{l j, Q_{j}-s-1}, \\
& \check{\Omega}_{r}(w)=\hat{L}(w)+\sum_{j} \sum_{s=0}^{Q_{j}-1} \frac{w^{s}}{s !} e^{-i \lambda_{j} w} \check{\Gamma}_{r j, Q_{j}-s-1} .
\end{aligned}
$$

\section{Symmetry Relations for General Potentials}

In this section we derive general symmetry relations on the Marchenko integral kernels and norming constant matrices which allow us to reduce the number of seemingly different Marchenko kernels from eight to four.

Introducing the partitioning

$$
\begin{aligned}
& {\left[\begin{array}{ll}
\bar{K}(x, y) & K(x, y)
\end{array}\right] }=\left[\begin{array}{ll}
K_{1}(x, y) & K_{2}(x, y) \\
K_{3}(x, y) & K_{4}(x, y)
\end{array}\right], \\
& {\left[\begin{array}{ll}
G(x, y) & \bar{G}(x, y)
\end{array}\right] }=\left[\begin{array}{ll}
G_{1}(x, y) & G_{2}(x, y) \\
G_{3}(x, y) & G_{4}(x, y)
\end{array}\right], \\
& {\left[\begin{array}{ll}
\check{K}(y, x) \\
\check{K}(y, x)
\end{array}\right]=\left[\begin{array}{cc}
\check{K}_{1}(y, x) & \check{K}_{2}(y, x) \\
\check{K}_{3}(y, x) & \check{K}_{4}(y, x)
\end{array}\right], \quad\left[\begin{array}{l}
\check{G}(y, x) \\
\breve{G}(y, x)
\end{array}\right]=\left[\begin{array}{ll}
\check{G}_{1}(y, x) & \check{G}_{2}(y, x) \\
\breve{G}_{3}(y, x) & \check{G}_{4}(y, x)
\end{array}\right], }
\end{aligned}
$$

we derive from (5.8) the following integral equations:

$$
\begin{gathered}
K_{3}(x, y)+\tilde{\Omega}_{l}(x+y)+\int_{x}^{\infty} d z K_{4}(x, z) \tilde{\Omega}_{l}(z+y)=0_{m \times n}, \\
G_{2}(x, y)+\tilde{\Omega}_{r}(x+y)+\int_{-\infty}^{x} d z G_{1}(x, z) \tilde{\Omega}_{r}(z+y)=0_{n \times m}, \\
G_{3}(x, y)+\Omega_{r}(x+y)+\int_{-\infty}^{x} d z G_{4}(x, z) \Omega_{r}(z+y)=0_{m \times n}, \\
K_{2}(x, y)+\Omega_{l}(x+y)+\int_{x}^{\infty} d z K_{1}(x, z) \Omega_{l}(z+y)=0_{n \times m}, \\
\check{G}_{2}(y, x)-\check{\Omega}_{r}(y+x)-\int_{-\infty}^{x} d z \check{\Omega}_{r}(y+z) \check{G}_{4}(z, x)=0_{n \times m}, \\
\check{K}_{3}(y, x)-\check{\Omega}_{l}(y+x)-\int_{x}^{\infty} d z \check{\Omega}_{l}(y+z) \check{K}_{1}(z, x)=0_{m \times n}, \\
\check{K}_{2}(y, x)-\check{\Omega}_{l}(y+x)-\int_{x}^{\infty} d z \check{\Omega}_{l}(y+z) \check{K}_{4}(z, x)=0_{n \times m}, \\
\check{G}_{3}(y, x)-\check{\Omega}_{r}(y+x)-\int_{-\infty}^{x} d z \check{\Omega}_{r}(y+z) \check{G}_{1}(z, x)=0_{m \times n} .
\end{gathered}
$$


We now derive the following proposition on recovering a Marchenko integral kernel from the matrix functions $K, G, \bar{K}$, and $\bar{G}$.

Proposition 6.1. For $y>x$ let $K(x, y), \check{K}(y, x), \mathscr{K}(x, y)$, and $\check{\mathscr{K}}(y, x)$ be measurable $p_{1} \times p_{2}$ matrix functions satisfying

$$
\begin{gathered}
\max \left(\int_{x}^{\infty} d y\|K(x, y)\|, \int_{x}^{\infty} d y\|\check{K}(y, x)\|\right)<1, \\
\int_{x}^{\infty} d y\|\mathscr{K}(x, y)\|+\int_{x}^{\infty} d y\|\check{\mathscr{K}}(y, x)\|<+\infty .
\end{gathered}
$$

Then the integral equations

$$
\begin{aligned}
& \Lambda(x+y)+\int_{x}^{\infty} d z K(x, z) \Lambda(z+y)=-\mathscr{K}(x, y), \\
& \check{\Lambda}(x+y)+\int_{x}^{\infty} d z \check{\Lambda}(z+y) \check{K}(z, x)=-\check{\mathscr{K}}(y, x),
\end{aligned}
$$

have unique measurable solutions $\Lambda(w)$ and $\check{\Lambda}(w)$ satisfying

$$
\int_{2 x}^{\infty} d w(\|\Lambda(w)\|+\|\tilde{\Lambda}(w)\|)<+\infty .
$$

A similar result is true for matrix functions depending on $(x, y)$ with $y<x$ and with integrals with respect to $y \in(-\infty, x)$.

Proof. Let us take the norm of the integral term in (6.3a) and integrate with respect to $y \in(x, \infty)$. Denoting the left-hand side of $(6.2)$ by $\delta \in[0,1)$, we obtain

$$
\begin{aligned}
\int_{x}^{\infty} d y \int_{x}^{\infty} d z\|K(x, z)\|\|\Lambda(z+y)\| & =\int_{x}^{\infty} d z \int_{x}^{z} d y\|K(x, z)\|\|\Lambda(z+y)\| \\
& \leqslant \delta \int_{2 x}^{\infty} d w\|\Lambda(w)\| .
\end{aligned}
$$

The proposition now follows from the contraction mapping principle.

In the scalar case $(n=m=1)$ the Jost matrices are unitary $2 \times 2$ matrices of determinant 1 for $\lambda \in \mathbb{R}$ and hence their inverses equal their cofactor matrices. Passing to the inverse Fourier transforms as in (5.4) we see that

$$
\begin{array}{lll}
\check{K}_{1}(y, x)=K_{4}(x, y), & \check{K}_{2}(y, x)=-K_{2}(x, y), \\
\check{K}_{3}(y, x)=-K_{3}(x, y), & \check{K}_{4}(y, x)=K_{1}(x, y), \\
\check{G}_{1}(y, x)=G_{4}(x, y), & \check{G}_{2}(y, x)=-G_{2}(x, y), \\
\check{G}_{3}(y, x)=-G_{3}(x, y), & \check{G}_{4}(y, x)=G_{1}(x, y) .
\end{array}
$$

Applying Proposition 6.1 to convert $(6.1 \mathrm{e})-(6.1 \mathrm{~h})$ to $(6.1 \mathrm{a})-(6.1 \mathrm{~d})$ on multiplication by a minus sign we obtain the symmetry relations

$$
\check{\Omega}_{l}(\alpha)=\Omega_{l}(\alpha), \quad \check{\Omega}_{r}(\alpha)=\Omega_{r}(\alpha), \quad \tilde{\Omega}_{l}(\alpha)=\tilde{\Omega}_{l}(\alpha), \quad \tilde{\Omega}_{r}(\alpha)=\tilde{\Omega}_{r}(\alpha) .
$$


This means that we can write all eight Marchenko integral equations in terms of only two Marchenko kernels $\Omega_{l}(\alpha)$ and $\Omega_{r}(\alpha)$. As a result we get

$$
\check{\Gamma}_{l j s}=\Gamma_{l j s}, \quad \check{\Gamma}_{r j s}=\Gamma_{r j s}, \quad \check{\Gamma}_{l j s}=\tilde{\Gamma}_{l j s}, \quad \check{\Gamma}_{r j s}=\tilde{\Gamma}_{r j s},
$$

where $s=0,1, \ldots, Q_{j}-1$ (in the first two) and $s=0,1, \ldots, \tilde{Q}_{j}-1$ (in the last two), respectively.

Let us now generalize the symmetry relations (6.4) and (6.5) to the general matrix Zakharov-Shabat system.

THEOREM 6.2. Under the technical hypothesis, suppose the entries of $q(x)$ and $r(x)$ belong to $L^{1}(\mathbb{R})$. Then the symmetry relations

$$
\check{\Omega}_{l}(\alpha)=\Omega_{l}(\alpha), \quad \check{\Omega}_{r}(\alpha)=\Omega_{r}(\alpha), \quad \tilde{\Omega}_{l}(\alpha)=\tilde{\Omega}_{l}(\alpha), \quad \tilde{\Omega}_{r}(\alpha)=\tilde{\Omega}_{r}(\alpha),
$$

are satisfied. Moreover,

$$
\check{\Gamma}_{l j s}=\Gamma_{l j s}, \quad \check{\Gamma}_{r j s}=\Gamma_{r j s}, \quad \check{\Gamma}_{l j s}=\tilde{\Gamma}_{l j s}, \quad \tilde{\Gamma}_{r j s}=\tilde{\Gamma}_{r j s},
$$

where $s=0,1, \ldots, Q_{j}-1$.

Proof. From $\Psi(\lambda, x) \check{\Psi}(\lambda, x)=I_{n+m}$ we get

$$
\Psi_{3}(\lambda, x) \check{\Psi}_{1}(\lambda, x)+\Psi_{4}(\lambda, x) \check{\Psi}_{3}(\lambda, x)=0_{m \times n} .
$$

Because of (5.1a), (5.1d), (5.1f), and (5.1g), this identity leads to the equality

$$
\begin{aligned}
& K_{3}(x, y)+\breve{K}_{3}(y, x) \\
& \quad+\int_{x}^{\infty} d z\left\{K_{3}(x, y+z-x) \check{K}_{1}(z, x)+K_{4}(x, z) \check{K}_{3}(z+y-x, x)\right\}=0_{m \times n},
\end{aligned}
$$

where $y>x$ (and hence $y+z-x>x$ whenever $z>x$ ). Adding the two integral equations (6.1a) and (6.1f) and using (6.1a), (6.1f), and (6.8) repeatedly we perform the following rather straightforward calculations:

$$
\begin{aligned}
0_{m \times n}= & K_{3}(x, y)+\check{K}_{3}(y, x)+\tilde{\Omega}_{l}(x+y)-\tilde{\Omega}_{l}(y+x) \\
& +\int_{x}^{\infty} d z K_{4}(x, z) \tilde{\Omega}_{l}(z+y)-\int_{x}^{\infty} d z \tilde{\Omega}_{l}(y+z) \check{K}_{1}(z, x) \\
= & -\int_{x}^{\infty} d z K_{3}(x, y+z-x) \check{K}_{1}(z, x) \\
& -\int_{x}^{\infty} d z K_{4}(x, z) \check{K}_{3}(z+y-x, x)+\tilde{\Omega}_{l}(x+y)-\tilde{\Omega}_{l}(y+x) \\
& +\int_{x}^{\infty} d z K_{4}(x, z) \tilde{\Omega}_{l}(z+y)-\int_{x}^{\infty} d z \tilde{\Omega}_{l}(y+z) \check{K}_{1}(z, x) \\
= & \tilde{\Omega}_{l}(x+y)-\tilde{\Omega}_{l}(y+x)+\int_{x}^{\infty} d z K_{4}(x, z)\left[\tilde{\Omega}_{l}(z+y)-\tilde{\Omega}_{l}(y+z)\right]
\end{aligned}
$$




$$
\begin{aligned}
& +\int_{x}^{\infty} d z\left[\tilde{\Omega}_{l}(z+y)-\tilde{\Omega}_{l}(y+z)\right] \check{K}_{1}(z, x) \\
& -\int_{x}^{\infty} d z\left[K_{3}(x, y+z-x)+\tilde{\Omega}_{l}(y+z)\right] \check{K}_{1}(z, x) \\
& -\int_{x}^{\infty} d z K_{4}(x, z)\left[\check{K}_{3}(z+y-x, x)-\tilde{\Omega}_{l}(z+y)\right] \\
= & \tilde{\Omega}_{l}(x+y)-\tilde{\Omega}_{l}(y+x)+\int_{x}^{\infty} d z K_{4}(x, z)\left[\tilde{\Omega}_{l}(z+y)-\tilde{\Omega}_{l}(y+z)\right] \\
+ & \int_{x}^{\infty} d z\left[\tilde{\Omega}_{l}(z+y)-\tilde{\Omega}_{l}(y+z)\right] \check{K}_{1}(z, x)+\int_{x}^{\infty} d z \int_{x}^{\infty} d w K_{4}(x, w) \\
& \times\left[\tilde{\Omega}_{l}(w+y+z-x)-\tilde{\Omega}_{l}(w+z+y-x)\right] K_{1}(w, x) .
\end{aligned}
$$

Using Proposition 6.1 we easily derive the unique solvability of this homogeneous integral equation for sufficiently large $x$, which implies that its solution $\tilde{\Omega}_{l}(x+y)-$ $\tilde{\Omega}_{l}(y+x)$ vanishes for sufficiently large $x$. Since in this solution the reflection coefficient terms cancel out, it follows with the help of (5.15a) and (5.15f) that $\tilde{\Omega}_{l}(x+y)-\tilde{\Omega}_{l}(y+x)$ is analytic in $x$ for every $y$ and hence vanishes identically. We have thus established the first of the identities (6.6). The other three identities (6.6) can be proved in a similar way.

\section{Symmetry Relations in the Focusing Case}

In the focusing case the potential $V(x)$ in (2.2) satisfies the symmetry relations

$$
V(x)^{\dagger}=-V(x) \text { or } \quad r(x)=q(x)^{\dagger} .
$$

Then the location and Jordan structure of the eigenvalues does not change under complex conjugation (cf. [14] for $n=m=1$, [13] for $n=1$ and $m=2$, and [3,11] in general), i.e., if $\lambda_{j}$ is an eigenvalue of $H$ in $\mathbb{C}^{+}$, then $\bar{\lambda}_{j}$ is an eigenvalue of $H$ in $\mathbb{C}^{-}$and vice versa, while the Hamiltonian $H$ has the same Jordan normal form associated with these two eigenvalues.

a. Transition, reflection and transmission matrices. The symmetry picture greatly simplifies in the focusing Zakharov-Shabat case where $n=m=1$. Then for $\lambda \in \mathbb{R}$ the transition matrices $A_{l}(\lambda)$ and $A_{r}(\lambda)$ are unitary $2 \times 2$ matrices of unit determinant which are each others inverses. Thus for $\lambda \in \mathbb{R}$ we have

$$
\begin{aligned}
{\left[\begin{array}{ll}
A_{r 1}(\lambda) & A_{r 2}(\lambda) \\
A_{r 3}(\lambda) & A_{r 4}(\lambda)
\end{array}\right] } & =\left[\begin{array}{cc}
A_{l 4}(\lambda) & -A_{l 2}(\lambda) \\
-A_{l 3}(\lambda) & A_{l 1}(\lambda)
\end{array}\right] \\
& =\left[\begin{array}{ll}
\overline{A_{l 1}(\lambda)} & \overline{A_{l 3}(\lambda)} \\
\overline{A_{l 2}(\lambda)} & \overline{A_{l 4}(\lambda)}
\end{array}\right]=\left[\begin{array}{ll}
\overline{A_{r 4}(\lambda)} & -\overline{A_{r 3}(\lambda)} \\
-\overline{A_{r 2}(\lambda)} & \overline{A_{r 1}(\lambda)}
\end{array}\right]
\end{aligned}
$$

where all of the entries are scalars. In view of (4.1) and (4.2) we then have

$$
T(\lambda) \stackrel{\text { def }}{=} T_{l}(\lambda)=T_{r}(\lambda)=\overline{\tilde{T}_{l}(\bar{\lambda})}=\overline{\tilde{T}_{r}(\bar{\lambda})}, \quad \lambda \in \overline{\mathbb{C}^{+}} \text {not a pole. }
$$


With the help of (4.3) and (4.4) we can then arrange the eigenvalues in $\mathbb{C}^{-}$in such a way that $\tilde{\lambda}_{j}=\bar{\lambda}_{j}$, so that $\tilde{Q}_{j}=Q_{j}$ and

$$
\tau_{l j s}=\tau_{r j s}=\overline{\tilde{\tau}_{l j s}}=\overline{\tilde{\tau}_{r j s}}, \quad s=0,1, \ldots, Q_{j}-1 .
$$

According to Corollary 3.2 all eigenvalues have geometric multiplicity 1 , but may have a nontrivial Jordan structure. Due to the unitarity of the Jost matrices, we have for $\lambda \in \mathbb{R}$

$$
\begin{array}{ll}
\Psi_{1}(\lambda, x)=\overline{\Psi_{4}(\lambda, x)}, & \Psi_{2}(\lambda, x)=-\overline{\Psi_{3}(\lambda, x)}, \\
\Phi_{1}(\lambda, x)=\overline{\Phi_{4}(\lambda, x)}, & \Phi_{2}(\lambda, x)=-\overline{\Phi_{3}(\lambda, x)}
\end{array}
$$

where we have used standard 1, 2,3,4 partitioning of Jost matrices.

In the focusing (generalized) Manakov case where $n=1$ and $m \geqslant 2$, the transition matrices $A_{l}(\lambda)$ and $A_{r}(\lambda)$ are unitary $(m+1) \times(m+1)$ matrices of unit determinant which are each others inverses. Thus for $\lambda \in \mathbb{R}$ we have

$$
A_{r 1}(\lambda)=\operatorname{det} A_{l 4}(\lambda)=\overline{A_{l 1}(\bar{\lambda})}=\overline{\operatorname{det} A_{r 4}(\bar{\lambda})},
$$

so that

$$
T_{l}(\lambda)=\operatorname{det} T_{r}(\lambda)=\overline{\tilde{T}_{l}(\bar{\lambda})}=\overline{\operatorname{det} \tilde{T}_{r}(\bar{\lambda})}, \quad \lambda \in \overline{\mathbb{C}^{+}} \text {not a pole. }
$$

Moreover,

$$
\begin{aligned}
A_{r 4}(\lambda) & =A_{l 4}(\bar{\lambda})^{\dagger}, & & \lambda \in \overline{\mathbb{C}^{+}}, \\
T_{r}(\lambda) & =\tilde{T}_{r}(\bar{\lambda})^{\dagger}, & & \lambda \in \overline{\mathbb{C}^{+}} \text {not a pole. }
\end{aligned}
$$

In the focusing matrix Zakharov-Shabat case $(n, m=1,2,3, \ldots)$ we obtain for $\lambda \in \overline{\mathbb{C}^{+}}$

$$
\begin{array}{rlrl}
A_{l 1}(\lambda) & =A_{r 1}(\bar{\lambda})^{\dagger}, & & T_{l}(\lambda)=\tilde{T}_{l}(\bar{\lambda})^{\dagger}, \\
A_{r 4}(\lambda) & =A_{l 4}(\bar{\lambda})^{\dagger}, & T_{r}(\lambda)=\tilde{T}_{r}(\bar{\lambda})^{\dagger}, \\
\operatorname{det} A_{r 1}(\lambda) & =\operatorname{det} A_{l 4}(\lambda)=\overline{\operatorname{det} A_{l 1}(\bar{\lambda})}=\overline{\operatorname{det} A_{r 4}(\bar{\lambda})} .
\end{array}
$$

According to Corollary 3.2 all eigenvalues have geometric multiplicity at $\operatorname{most} \min (n, m)$. Moreover,

$$
\tau_{l j s}=\tilde{\tau}_{l j s}^{\dagger}, \quad \tau_{r j s}=\tilde{\tau}_{r j s}^{\dagger}, \quad s=0,1, \ldots, Q_{j}-1 .
$$

Using (5.1) and (2.10) we now derive the symmetry relations

$$
\begin{array}{lll}
K(x, y)=\overline{\check{K}}(y, x)^{\dagger}, & \bar{K}(x, y)=\check{K}(y, x)^{\dagger}, & y>x, \\
G(x, y)=\bar{G}(y, x)^{\dagger}, & \bar{G}(x, y)=\check{G}(y, x)^{\dagger}, & y<x .
\end{array}
$$

In the focusing case the scattering matrix is $J$-unitary in the sense that

$$
S(\lambda)^{\dagger}=J S(\lambda)^{-1} J=J \tilde{S}(\lambda) J, \quad \lambda \in \mathbb{R},
$$


which implies (7.1a) and (7.1b) as well as

$$
\tilde{R}(\lambda)=-R(\lambda)^{\dagger}, \quad \tilde{L}(\lambda)=-L(\lambda)^{\dagger} .
$$

As a result of (7.4) and (4.13) we get

$$
\hat{\tilde{R}}(\alpha)=-\hat{R}(\alpha)^{\dagger}, \quad \hat{\tilde{L}}(\alpha)=-\hat{L}(\alpha)^{\dagger}, \quad \alpha \in \mathbb{R} .
$$

Thus, as indicated by (7.3) and (7.5), one converts the Marchenko equations (5.2a), (5.2b), (5.2c), and (5.2d) into the respective Marchenko equations (5.2g), (5.2h), (5.2e), and (5.2f) by taking the conjugate transpose.

b. Recovering Marchenko integral kernels from Jost solutions. Our goal is to generalize the symmetry relations (7.5) as follows:

THEOREM 7.1. Under the technical hypothesis, suppose the entries of $q(x)$ and $r(x)=q(x)^{\dagger}$ belong to $L^{1}(\mathbb{R})$. Then the symmetry relations:

$$
\begin{array}{ll}
\tilde{\Omega}_{l}(\alpha)=-\check{\Omega}_{l}(\alpha)^{\dagger}, & \tilde{\Omega}_{r}(\alpha)=-\check{\Omega}_{r}(\alpha)^{\dagger}, \\
\Omega_{l}(\alpha)=-\check{\Omega}_{l}(\alpha)^{\dagger}, & \Omega_{r}(\alpha)=-\check{\Omega}_{r}(\alpha)^{\dagger},
\end{array}
$$

are satisfied.

Proof. Because of (7.5) it is sufficient to prove the symmetry relations (7.6) for the bound state contributions to the Marchenko integral equations. But these are analytic functions of their independent variable. Moreover, choose a sufficiently large $x$ such that

$$
\max _{j=1,4}\left[\max \left(\int_{x}^{\infty} d y\left\|K_{j}(x, y)\right\|, \int_{x}^{\infty} d y\left\|\breve{K}_{j}(y, x)\right\|\right)\right]<1 .
$$

Then Proposition 6.1 implies the unique solvability of (6.1a), (6.1d), (6.1f), and (6.1g) for large $x$. Since, apart from a minus sign, the conjugate transposes of (6.1a) and (6.1d) coincide with $(6.1 \mathrm{~g})$ and $(6.1 \mathrm{f})$, respectively, the symmetry relations (7.6) follow for large $x$. In the same way we can prove the symmetry relations right for sufficiently negative $x$. Analytic continuation of the bound state contributions will do the rest.

If the resolvent of the Hamiltonian has only simple poles, from (5.9) we now derive the following symmetry relations for the norming constant matrices:

$$
\begin{array}{ll}
\Gamma_{l j 0}=-\left(\check{\Gamma}_{l j 0}\right)^{\dagger}, & \Gamma_{r j 0}=-\left(\tilde{\Gamma}_{r j 0}\right)^{\dagger}, \\
\tilde{\Gamma}_{l j 0}=-\left(\check{\Gamma}_{l j 0}\right)^{\dagger}, & \tilde{\Gamma}_{r j 0}=-\left(\check{\Gamma}_{r j 0}\right)^{\dagger} .
\end{array}
$$

For general Jordan structure we obtain from (5.15)

$$
\begin{array}{ll}
\Gamma_{l j s}=-\left(\check{\Gamma}_{l j s}\right)^{\dagger}, & \Gamma_{r j s}=-\left(\check{\Gamma}_{r j s}\right)^{\dagger}, \\
\tilde{\Gamma}_{l j s}=-\left(\check{\Gamma}_{l j s}\right)^{\dagger}, & \tilde{\Gamma}_{r j s}=-\left(\check{\Gamma}_{r j s}\right)^{\dagger},
\end{array}
$$

where $s=0,1, \ldots, Q_{j}-1$.

Summarizing we have proved the following result: 
THEOREM 7.2. Under the technical hypothesis, suppose the entries of $q(x)$ and $r(x)=q(x)^{\dagger}$ belong to $L^{1}(\mathbb{R})$. Then the symmetry relations

$$
\begin{aligned}
& \tilde{\Omega}_{l}(\alpha)=\tilde{\Omega}_{l}(\alpha)=-\Omega_{l}(\alpha)^{\dagger}=-\check{\Omega}_{l}(\alpha)^{\dagger}, \\
& \tilde{\Omega}_{r}(\alpha)=\tilde{\Omega}_{r}(\alpha)=-\Omega_{r}(\alpha)^{\dagger}=-\check{\Omega}_{r}(\alpha)^{\dagger},
\end{aligned}
$$

are satisfied. Moreover,

$$
\begin{gathered}
\Gamma_{l j s}=\check{\Gamma}_{l j s}=-\left(\tilde{\Gamma}_{l j s}\right)^{\dagger}=-\left(\tilde{\check{\Gamma}}_{l j s}\right)^{\dagger}, \\
\Gamma_{r j s}=\check{\Gamma}_{r j s}=-\left(\tilde{\Gamma}_{r j s}\right)^{\dagger}=-\left(\check{\Gamma}_{r j s}\right)^{\dagger},
\end{gathered}
$$

where $s=0,1, \ldots, Q_{j}-1$.

\section{Acknowledgments}

The research leading to this article was supported in part by the Italian Ministry of Education and Research (MIUR) under PRIN grant no. 2006017542-003, and by INdAM-GNCS.

\section{REFERENCES}

[1] M.J. Ablowitz AND P.A. Clarkson, Solitons, Nonlinear Evolution Equations and Inverse Scattering, Cambridge University Press, Cambridge, 1991.

[2] M.J. Ablowitz, D.J. KAUP, A.C. NewELl, AND H. SEgur, The inverse scattering transform - Fourier analysis for nonlinear problems, Stud. Appl. Math. 53, 249-315 (1974).

[3] M.J. AblowitZ, B. PrinaRI, AND A.D. TRuBATCH, Discrete and Continuous Nonlinear Schrödinger Systems, Cambridge University Press, Cambridge, 2004.

[4] M.J. ABLowitz AND H. SEgUR, Solitons and the Inverse Scattering Transform, SIAM, Philadelphia, 1981.

[5] T. AKTOSUn, M. Klaus, AND C. VAN DER MEE, Wave scattering in one dimension with absorption, J. Math. Phys. 39, 1957-1992 (1998).

[6] T. AKTOSUn, M. KLAUS, AND C. VAN DER MeE, Direct and inverse scattering for selfadjoint Hamiltonian systems on the line, Integral Equations and Operator Theory 38, 129-171 (2000).

[7] T. AKTOSUn, M. KLAUS, AND C. VAN DER MEE, Small-energy asymptotics of the scattering matrix for the matrix Schrödinger equation on the line, J. Math. Phys. 42, 4627-4652 (2001).

[8] K. ChadAN And P. SABATIER, Inverse Problems in Quantum Scattering Theory, 2nd ed., Springer, New York, 1989.

[9] E.A. CodDInGTON AND N. Levinson, Theory of Ordinary Differential Equations, International Series in Pure and Applied Mathematics, McGraw-Hill, New York, 1955.

[10] P. DeIFT AND E. TRUBOWITZ, Inverse scattering on the line, Commun. Pure Appl. Math. 32, 121-251 (1979).

[11] F. DEMOnTIS, Direct and Inverse Scattering of the Matrix Zakharov-Shabat System, Ph.D. thesis, University of Cagliari, Italy, 2007.

[12] L.D. FADDEEV, Properties of the $S$-matrix of the one-dimensional Schrödinger equation, Amer. Math. Soc. Transl. 2, 139-166 (1964); also: Trudy Mat. Inst. Steklova 73, 314-336 (1964) [Russian].

[13] M. KLAUS, Remarks on the eigenvalues of the Manakov system, Mathematics and Computers in Simulation 69, 356-367 (2005).

[14] M. KLAus AND J.K. SHaW, On the eigenvalues of Zakharov-Shabat systems, SIAM J. Math. Anal. 34(4), 759-773 (2003).

[15] S.V. MANAKOV, On the theory of two-dimensional stationary self-focusing of electromagnetic waves, Sov. Phys. JETP 38, 248-253 (1974). 
[16] S. TANAKA, Non-linear Schrödinger equation and modified Korteweg-de Vries equation; Construction of solutions in terms of scattering data, Publ. Res. Inst. Math. Sci. 10, 329-357 (1974/75).

[17] C. VAN DER MEE Direct and inverse scattering for skewselfadjoint Hamiltonian systems. In: J.A. Ball, J.W. Helton, M. Klaus, and L. Rodman (eds.), Current Trends in Operator Theory and its Applications, Birkhäuser OT 149, Basel and Boston, 2004, pp. 407-439.

[18] V.E. ZAKHAROV AND A.B. SHABAT, Exact theory of two-dimensional self-focusing and one dimensional self-modulation of waves in nonlinear media, Sov. Phys. JETP 34, 62-69 (1972).

(Received July 12, 2007)

Francesco Demontis Dip. Matematica e Informatica Università di Cagliari Viale Merello 92 09123 Cagliari Italy

e-mail: fdemontis@unica.it

Cornelis van der Mee Dip. Matematica e Informatica Università di Cagliari Viale Merello 92 09123 Cagliari Italy

e-mail: cornelis@krein.unica.it 